Supplementary Material

\title{
Comparative studies of structures and peroxidase-like activities of copper(II) and iron(III) complexes with an EDTA-based phenylene- macrocycle and its acyclic analogue
}

\author{
Melissa Beltran-Torres, ${ }^{1}$ Rocío Sugich-Miranda, ${ }^{* 2}$ Hisila Santacruz-Ortega, ${ }^{* 1}$ Lorena Machi, ${ }^{1}$ \\ Motomichi Inoue, ${ }^{1}$ Enrique F. Velázquez, ${ }^{2}$ Yedith Soberanes, ${ }^{1,3}$ Herbert Höpfl, ${ }^{4}$ Refugio Pérez \\ González, ${ }^{1}$ Rosa Elena Navarro, ${ }^{1}$ Alex Salazar Medina, ${ }^{1}$ and Rogerio R. Sotelo Mundo ${ }^{3}$ \\ 1 Departamento de Investigación en Polímeros y Materiales. Universidad de Sonora. Blvd. Luis Encinas y \\ Calle Rosales S/N. Hermosillo, Sonora, 83000, México. \\ 2 Departamento de Ciencias Químico Biológicas. Universidad de Sonora. Blvd. Luis Encinas y Calle Rosales \\ S/N. Hermosillo, Sonora, 83000, México. \\ 3 Centro de Investigación en Alimentación y Desarrollo A.C. (CIAD) Carretera Gustavo Enrique Astiazarán \\ Rosas Num.46, Col. La Victoria, Hermosillo, Sonora, 83304, México. \\ 4 Centro de Investigaciones Químicas, Instituto de Investigación en Ciencias Básicas y Aplicadas. \\ Universidad Autónoma del Estado de Morelos. Av. Universidad 1001, Cuernavaca, Morelos, 62209, México. \\ * rocio.sugich@unison.mx, hisila.santacruz@unison.mx
}

Characterization by ESI-MS

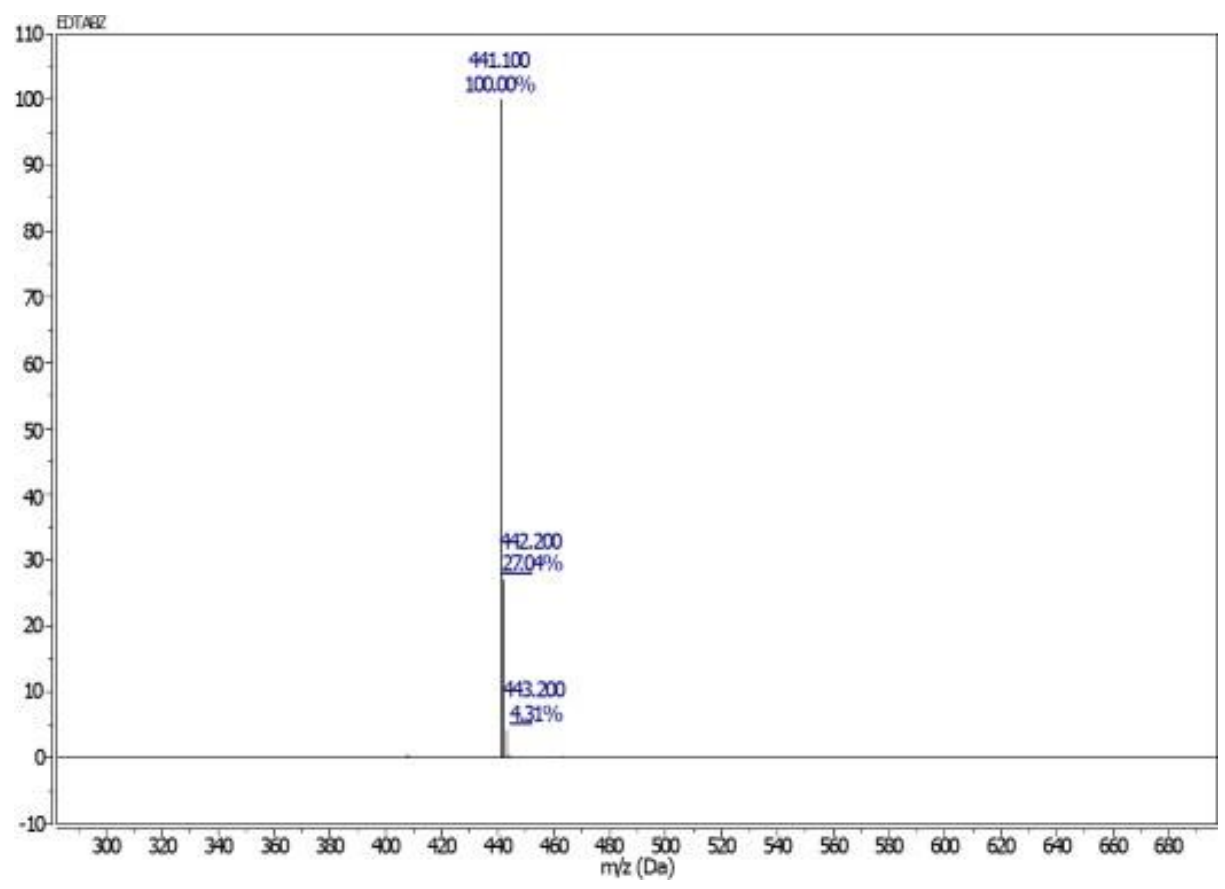

Figure S1. Mass Spectrum of edtabzH $\mathrm{H}_{2}$ 


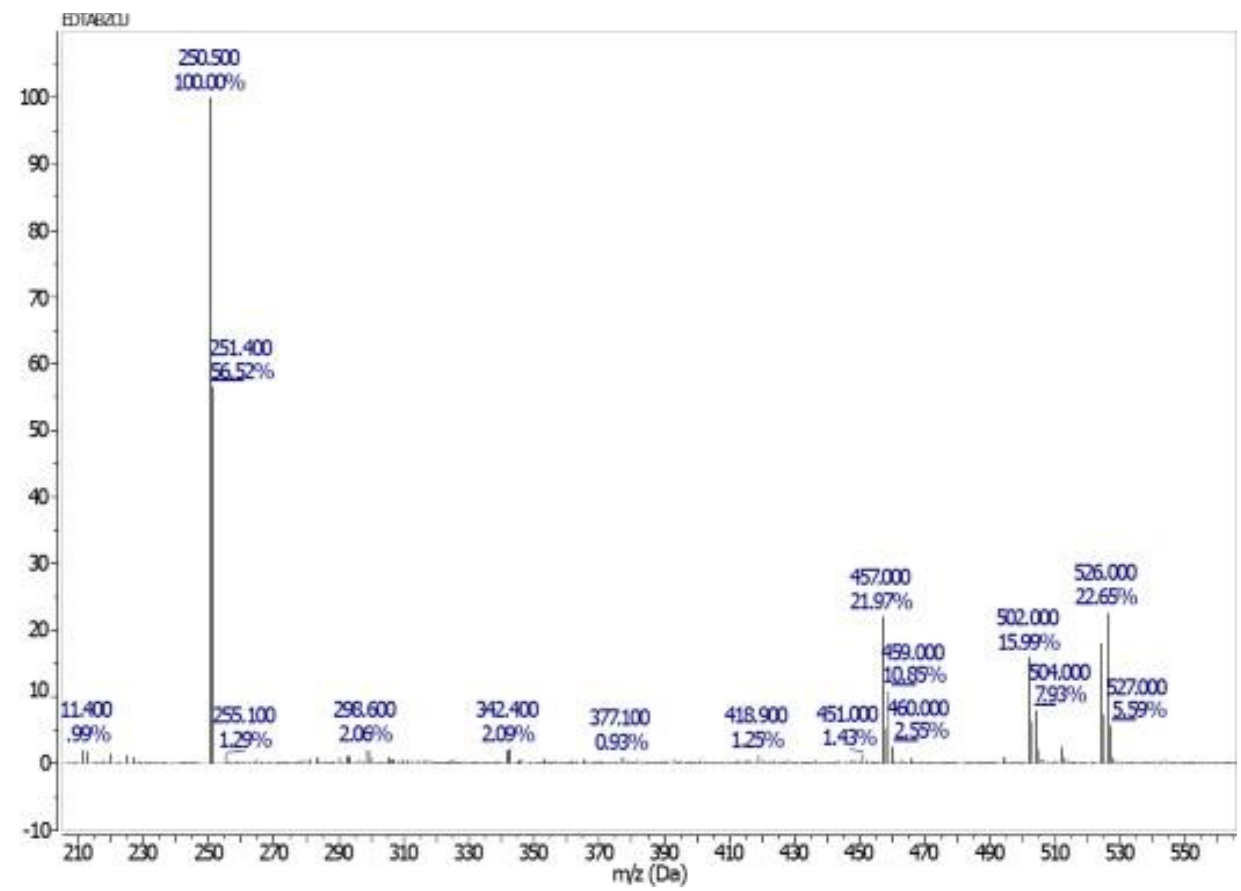

Figure S2. Mass Spectrum of [Cuedtabz]

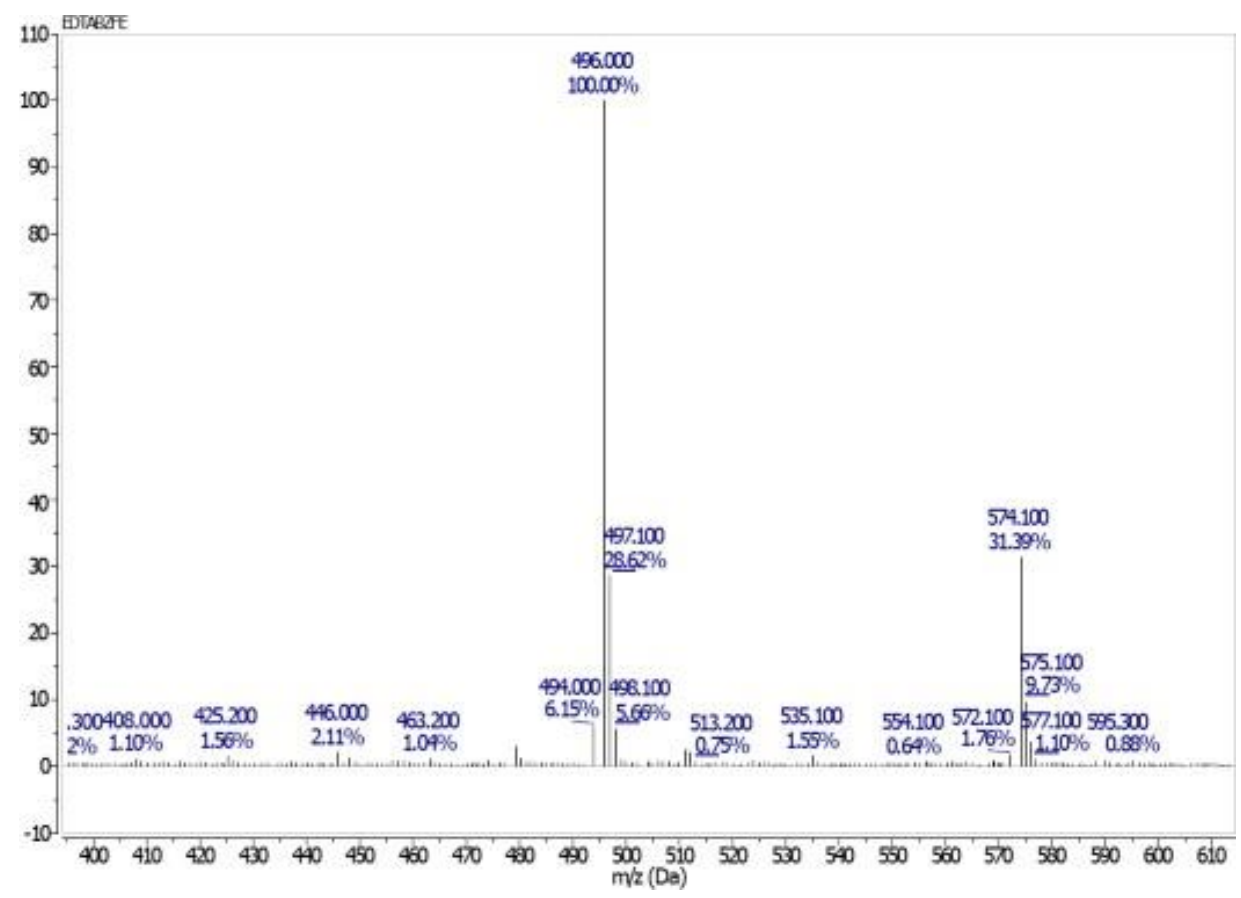

Figure S3. Mass Spectrum of [Feedtabz] 


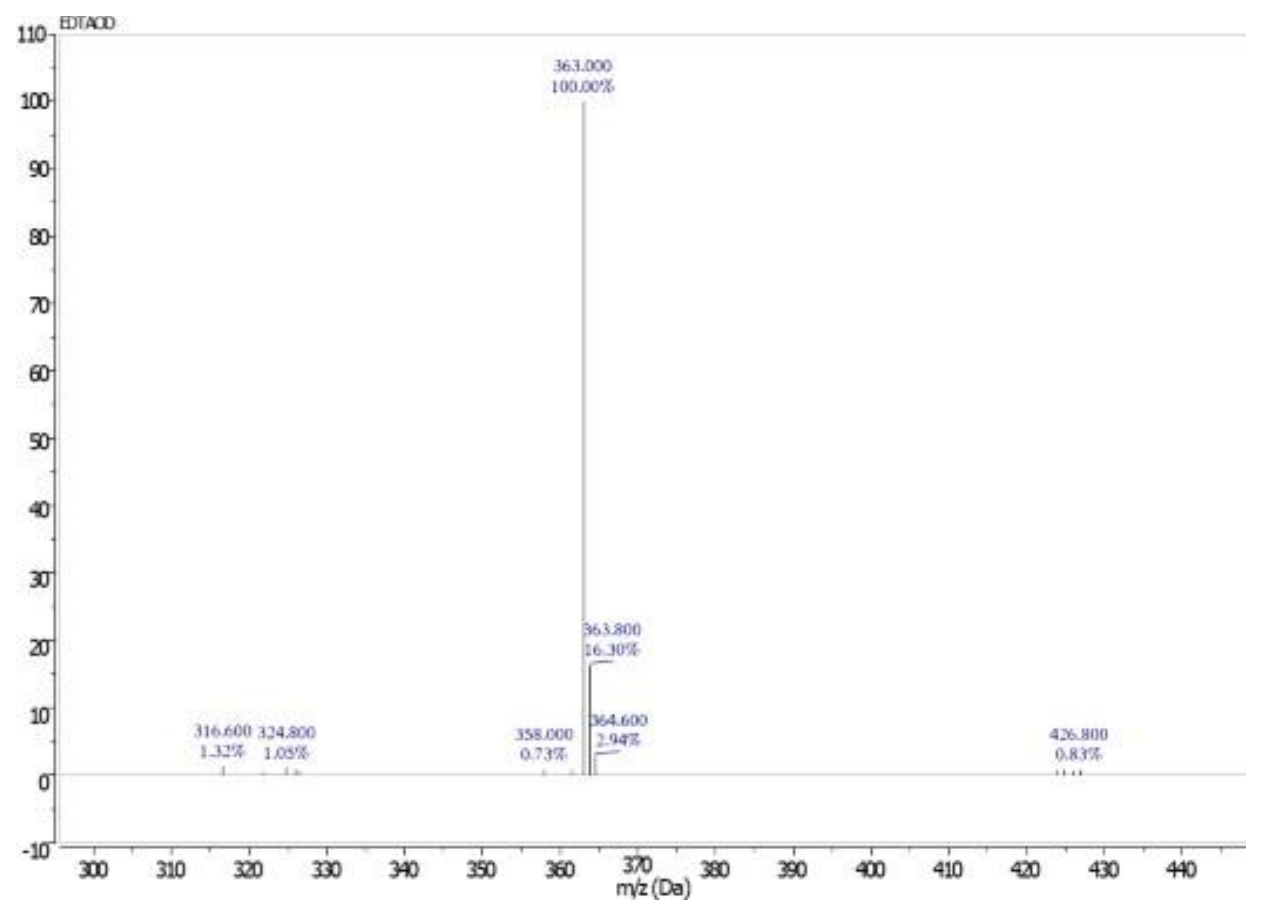

Figure S4. Mass Spectrum of edtaodH ${ }_{2}$

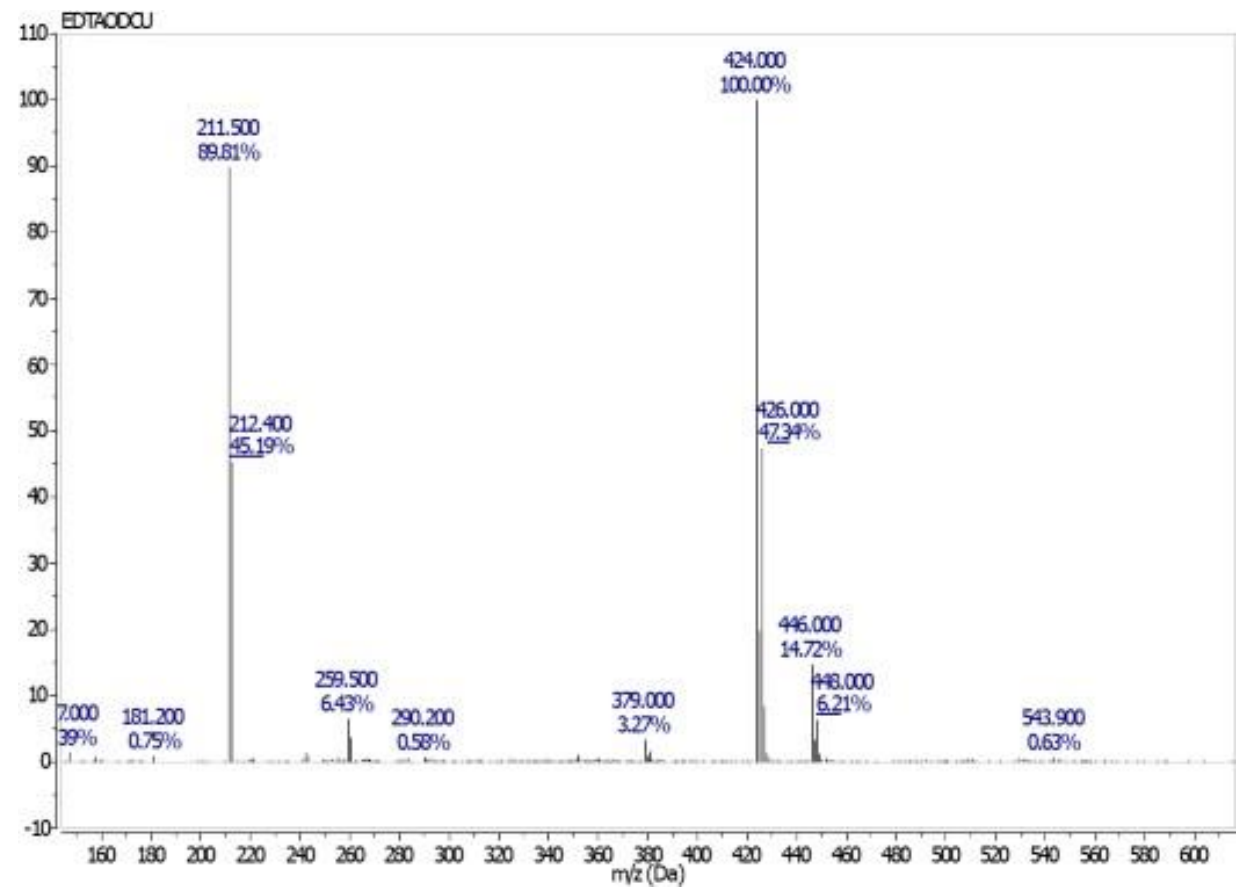

Figure S5. Mass Spectrum of [Cuedtaod] 


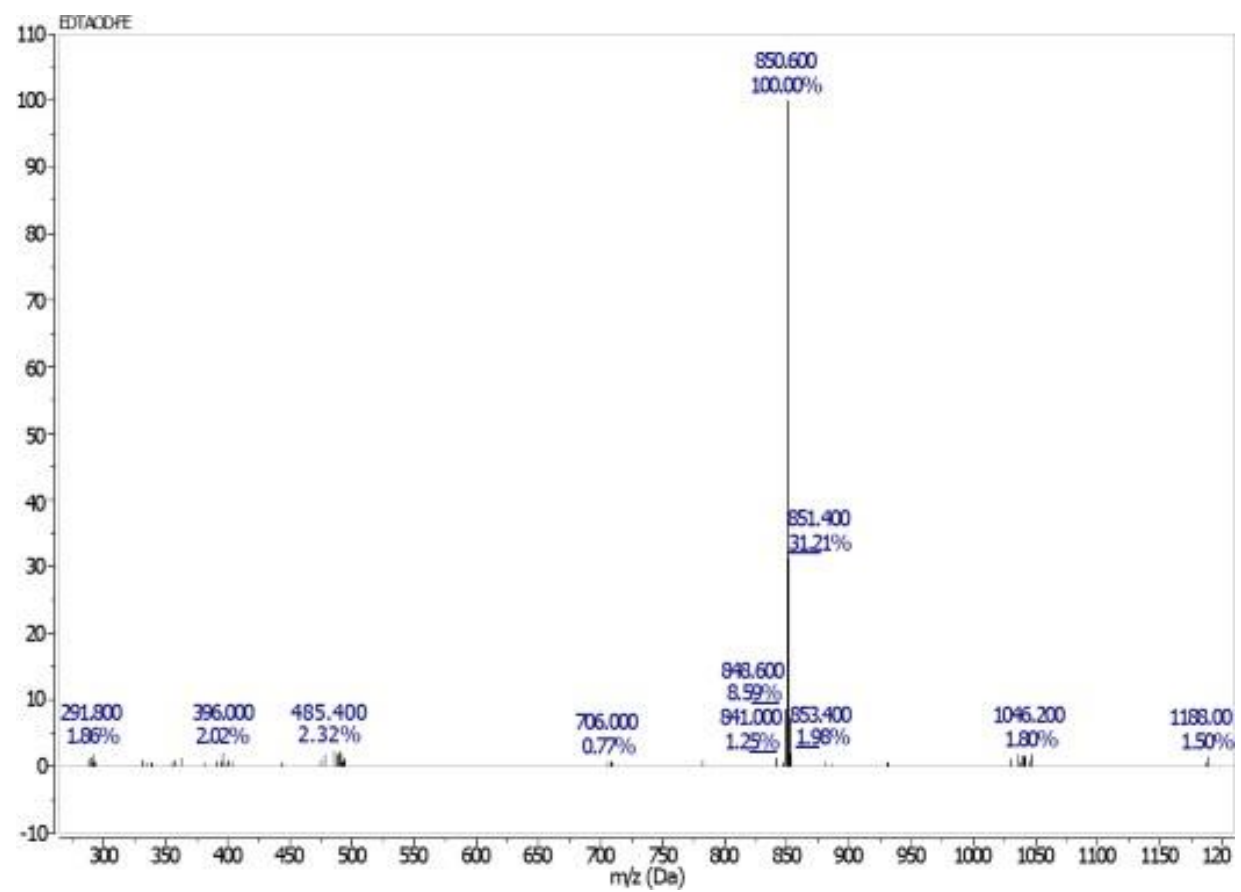

Figure S6. Mass Spectrum of $\left\{[\text { Feedtaod }]_{2} \mathrm{O}\right\}$

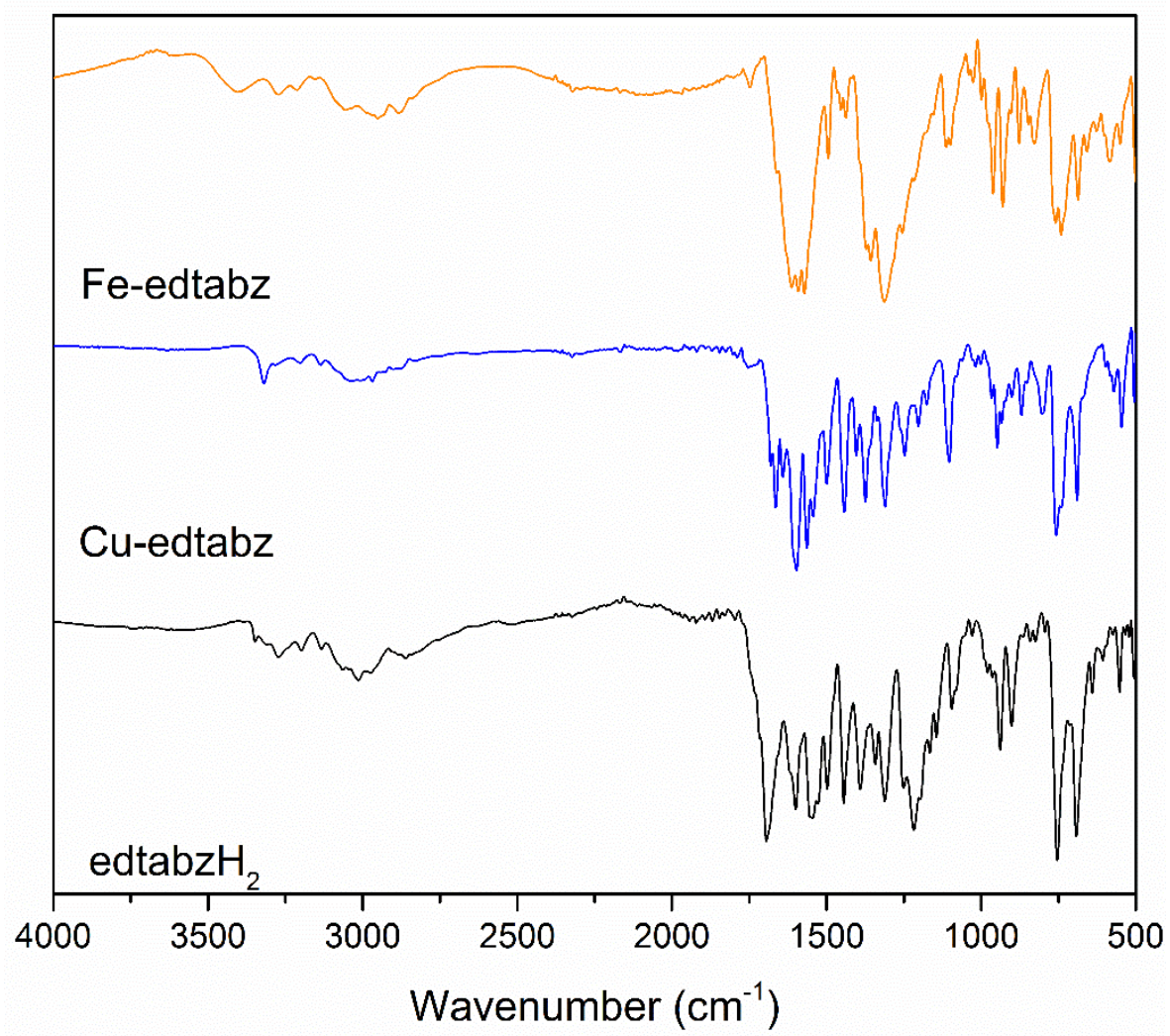

Figure S7. FTIR spectra in $\mathrm{KBr}$ of edtabz and complexes 


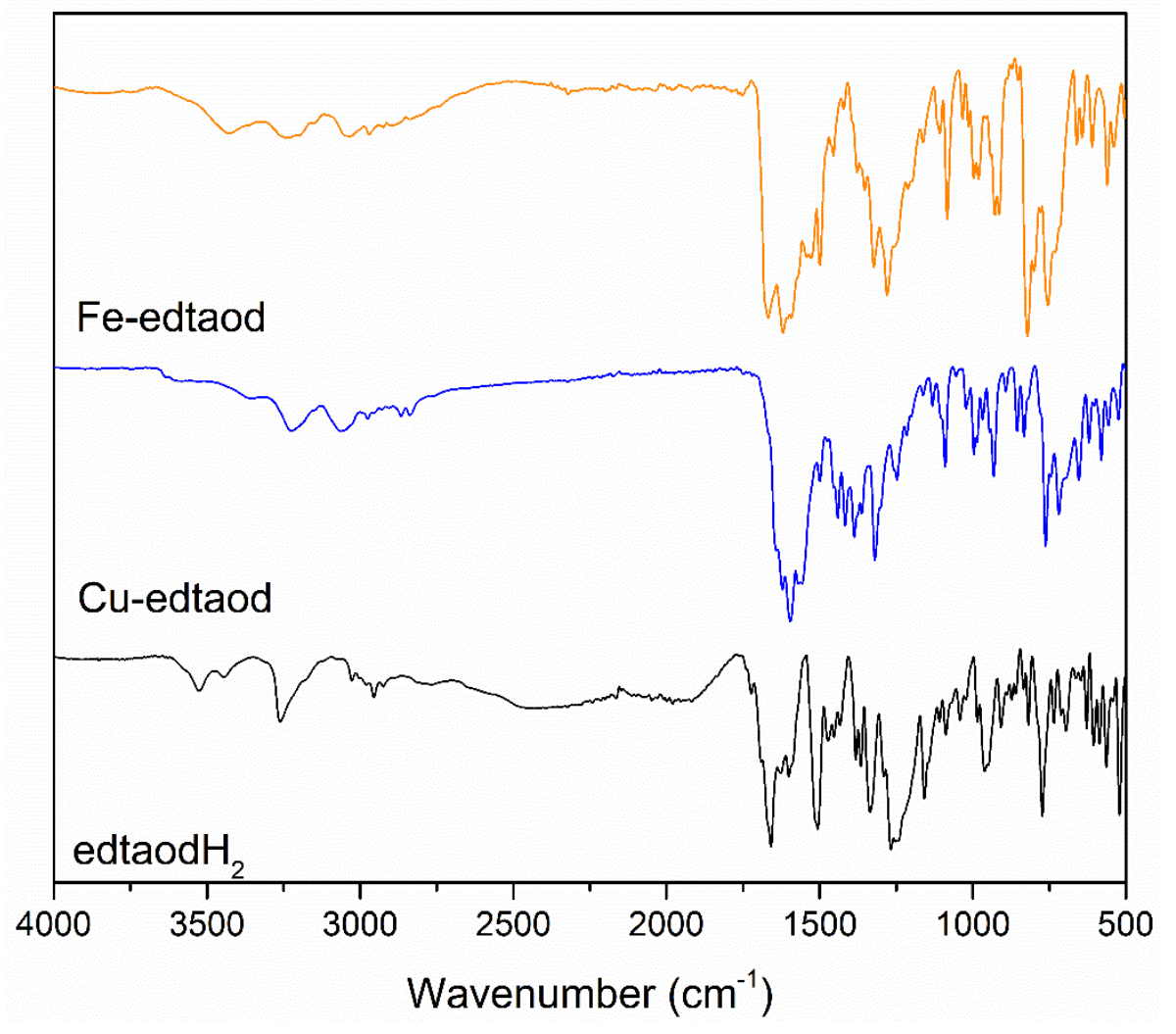

Figure S8. FTIR spectra in $\mathrm{KBr}$ of edtabz and complexes 


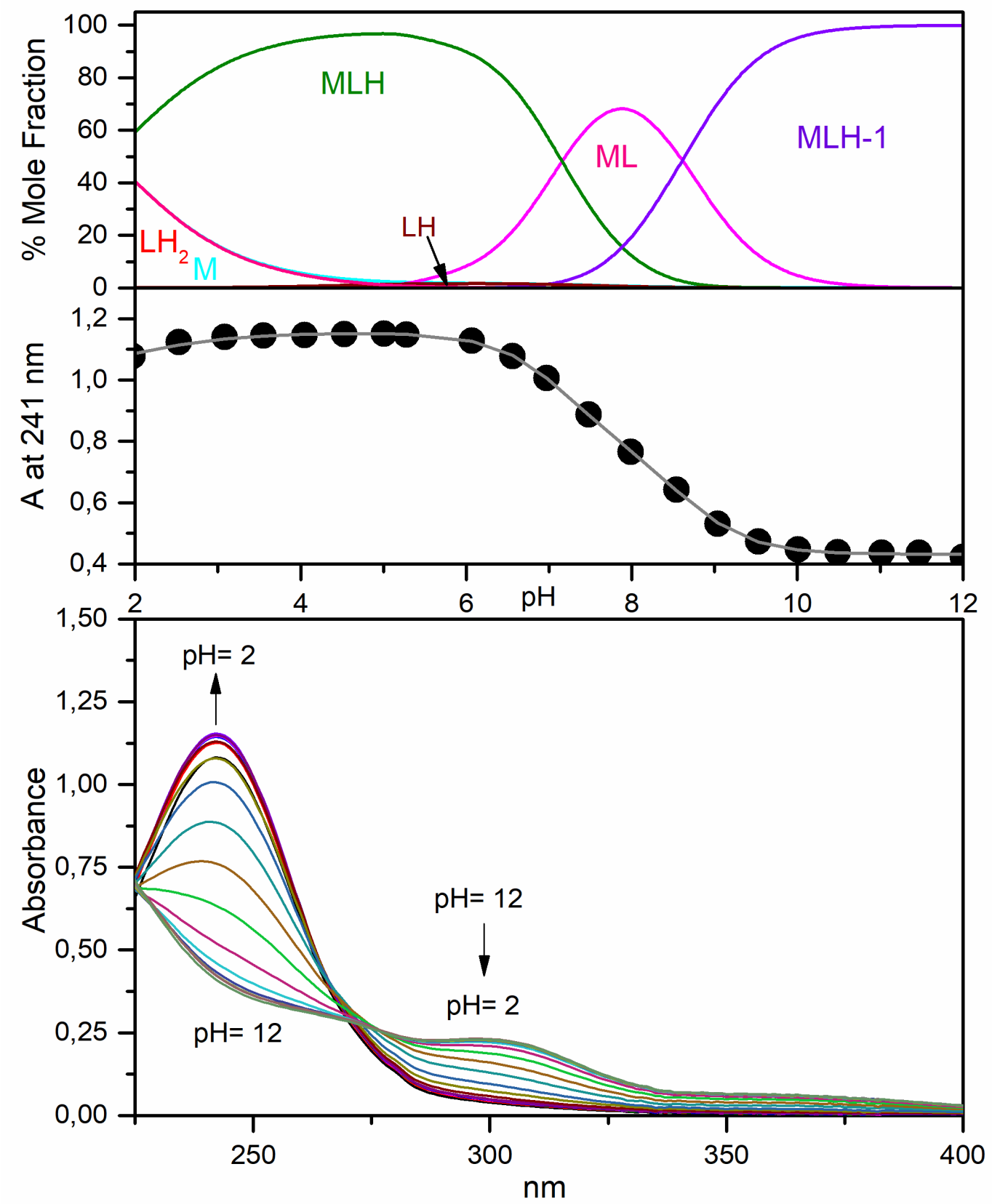

Figure S9. (Bottom) UV-Vis spectra of edtabz-Cu at different $\mathrm{pH}$ values. (Middle) variation of Absorbance at $241 \mathrm{~nm}$ at different $\mathrm{pH}$ values. (Top) species formation at different $\mathrm{pH}$ values. 


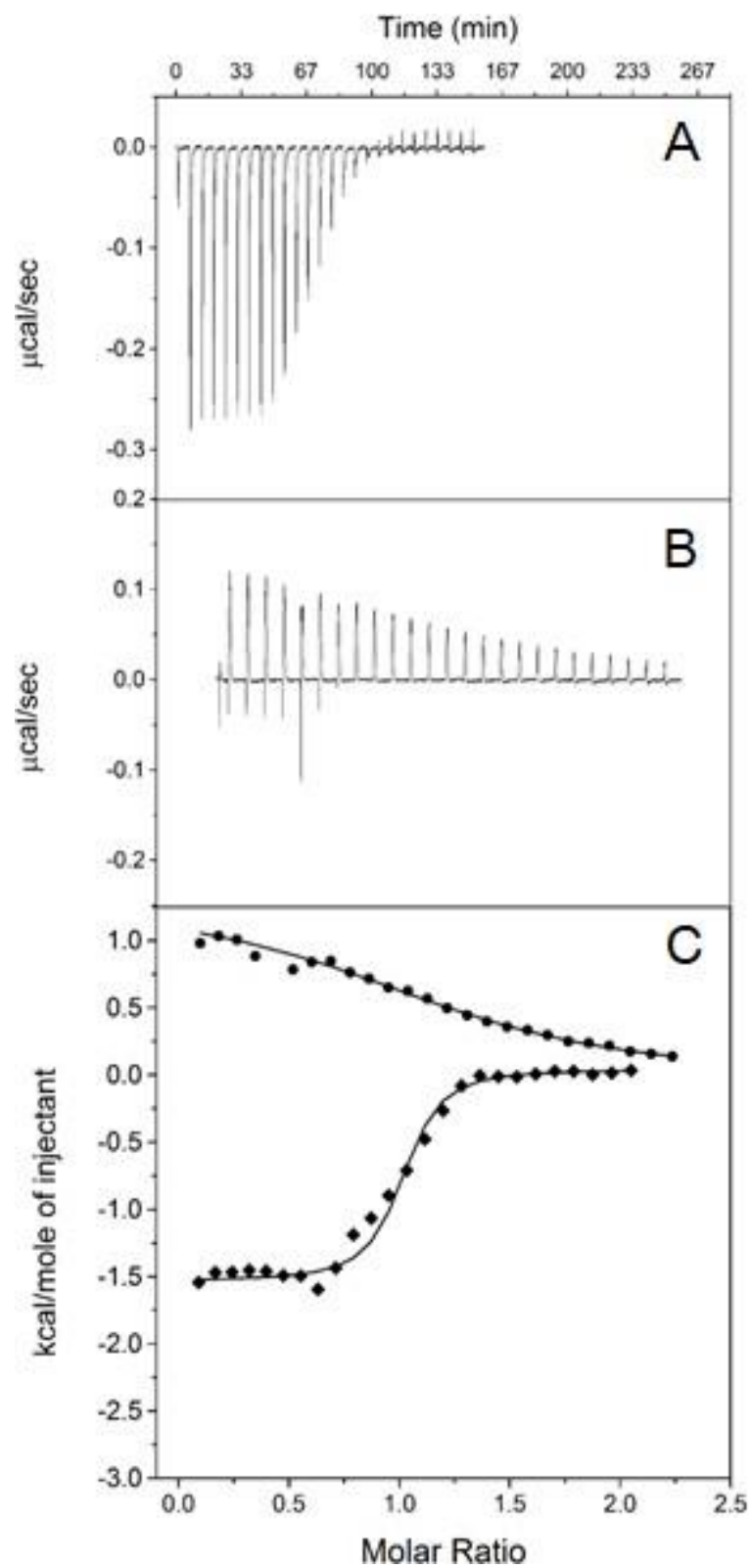

Figure S10. Microcalorimetric titrations of the edtaod ligand with $\mathrm{Cu}\left(\mathrm{NO}_{3}\right)_{2}$ and $\mathrm{Fe}\left(\mathrm{NO}_{3}\right)_{3}$ (in potassium hydrogen phthalate buffer at $0.5 \mathrm{M} \mathrm{pH} 3.0$ at $T 298.15 \mathrm{~K}$ ). The raw data of the ITC experiment are shown in the upper panels: (A) raw data of the system [Cu(edtaod)]; (B) raw data of the system [Fe(edtaod)]. Panel (C) shows the integrated data and curves fitted by AFFINIMETER. The rhomboid shapes correspond to the [Cu(edtaod)] system and the circles to the [Fe(edtaod)] system. 


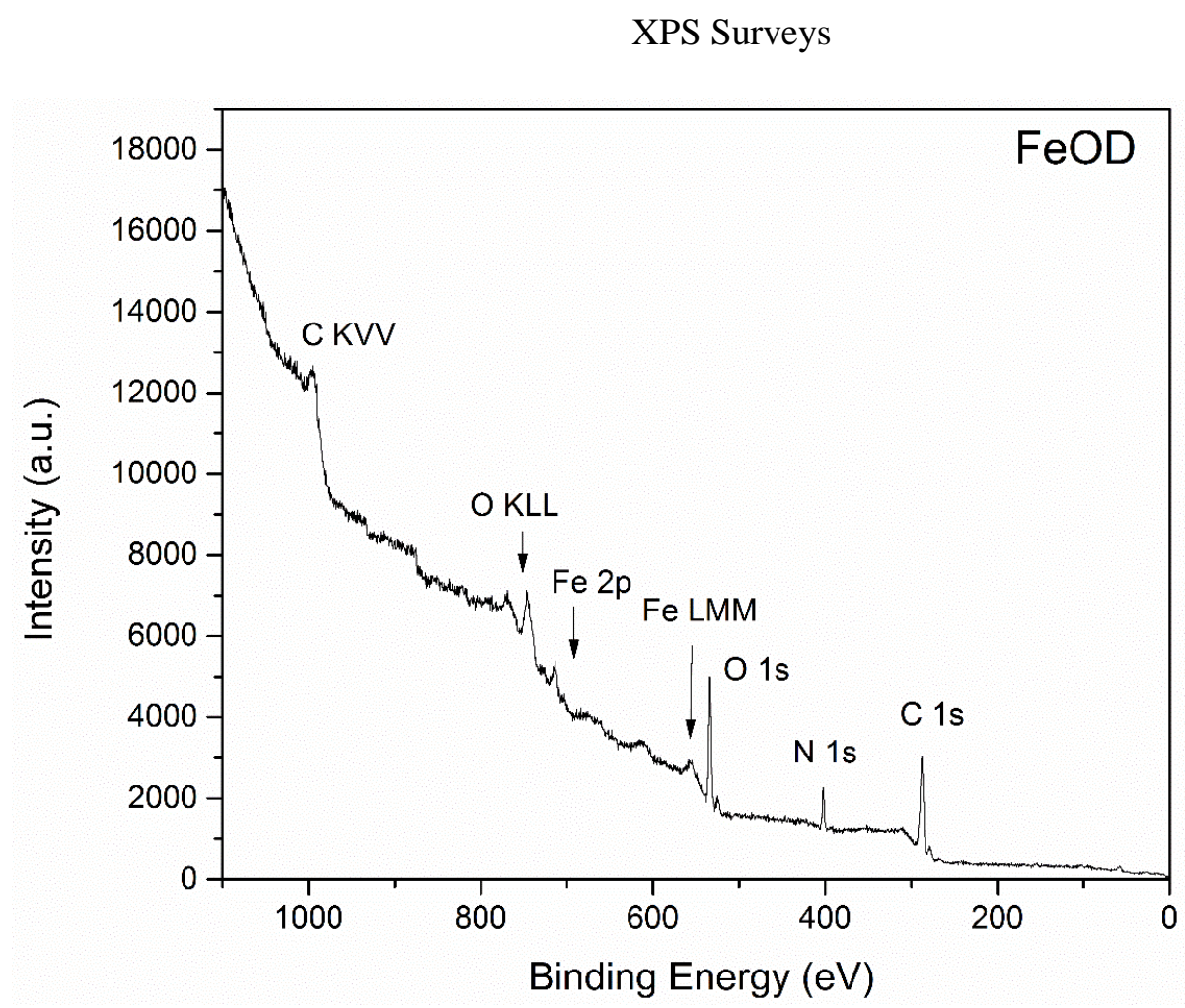

Figure S11. XPS of Feedtaod

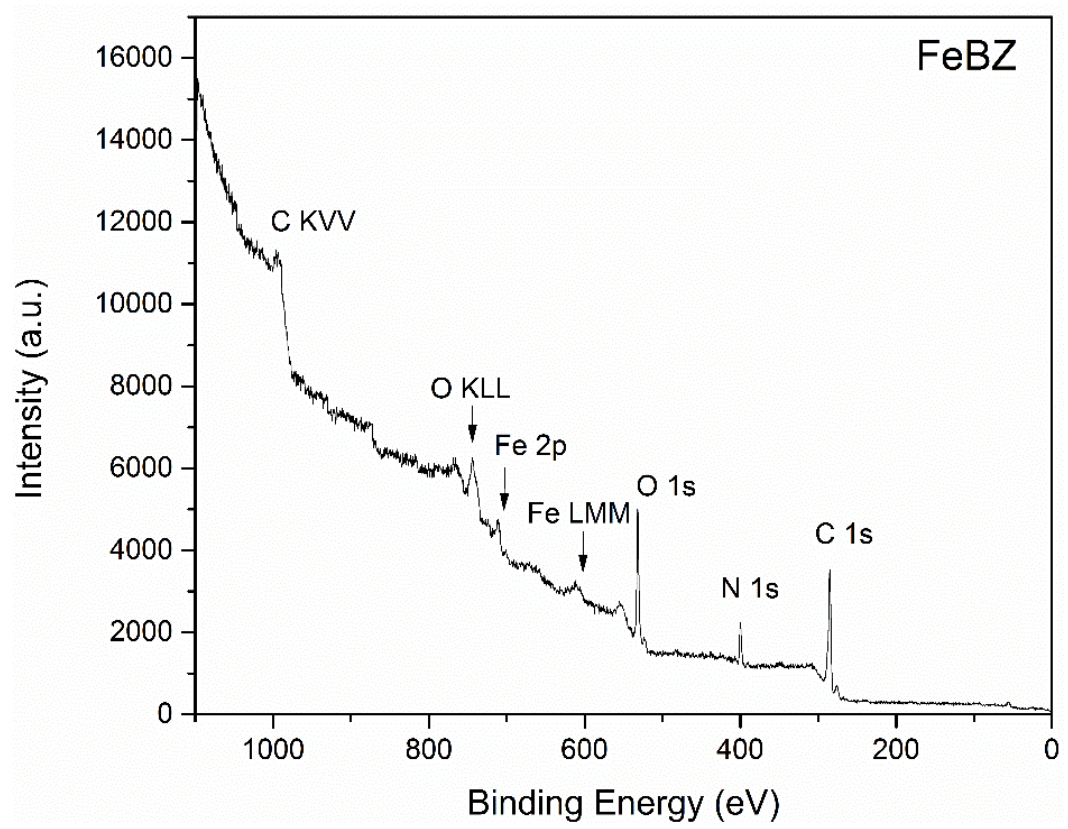

Figure S12. XPS of Feedtabz 


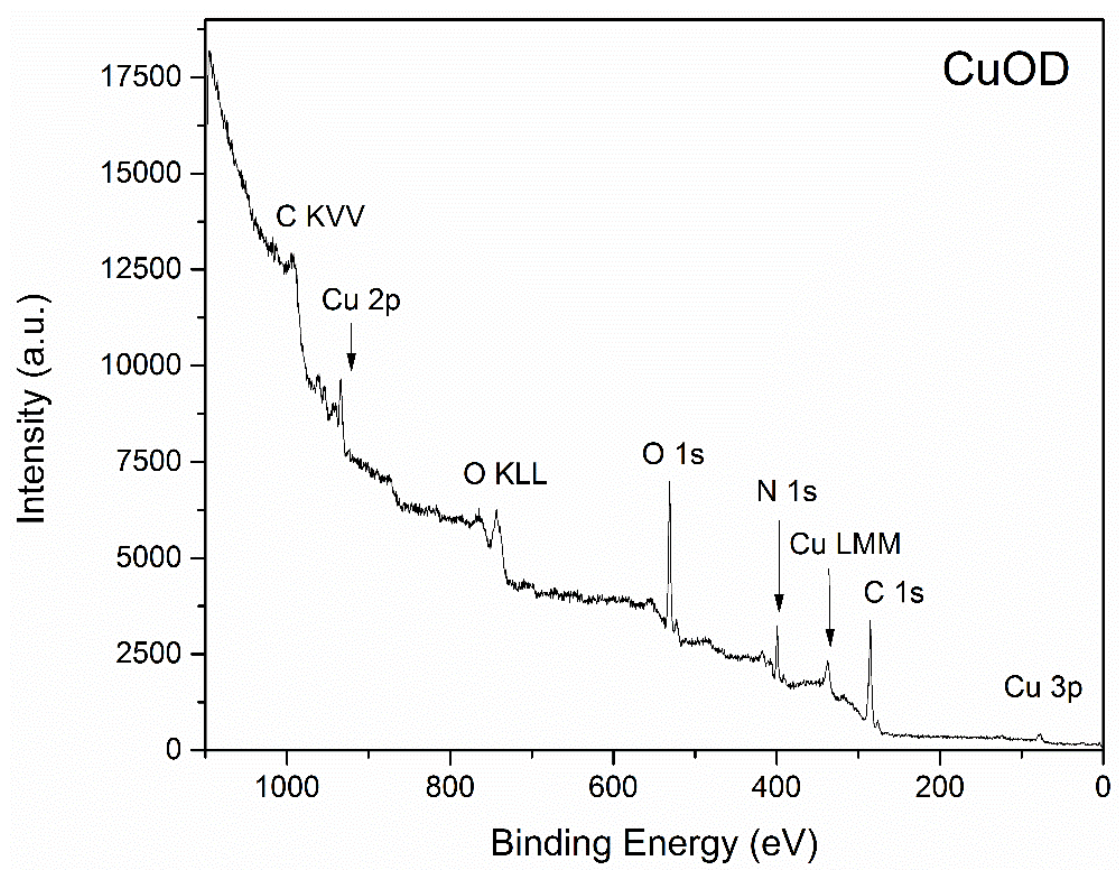

Figure S13. XPS of Cuedtaod

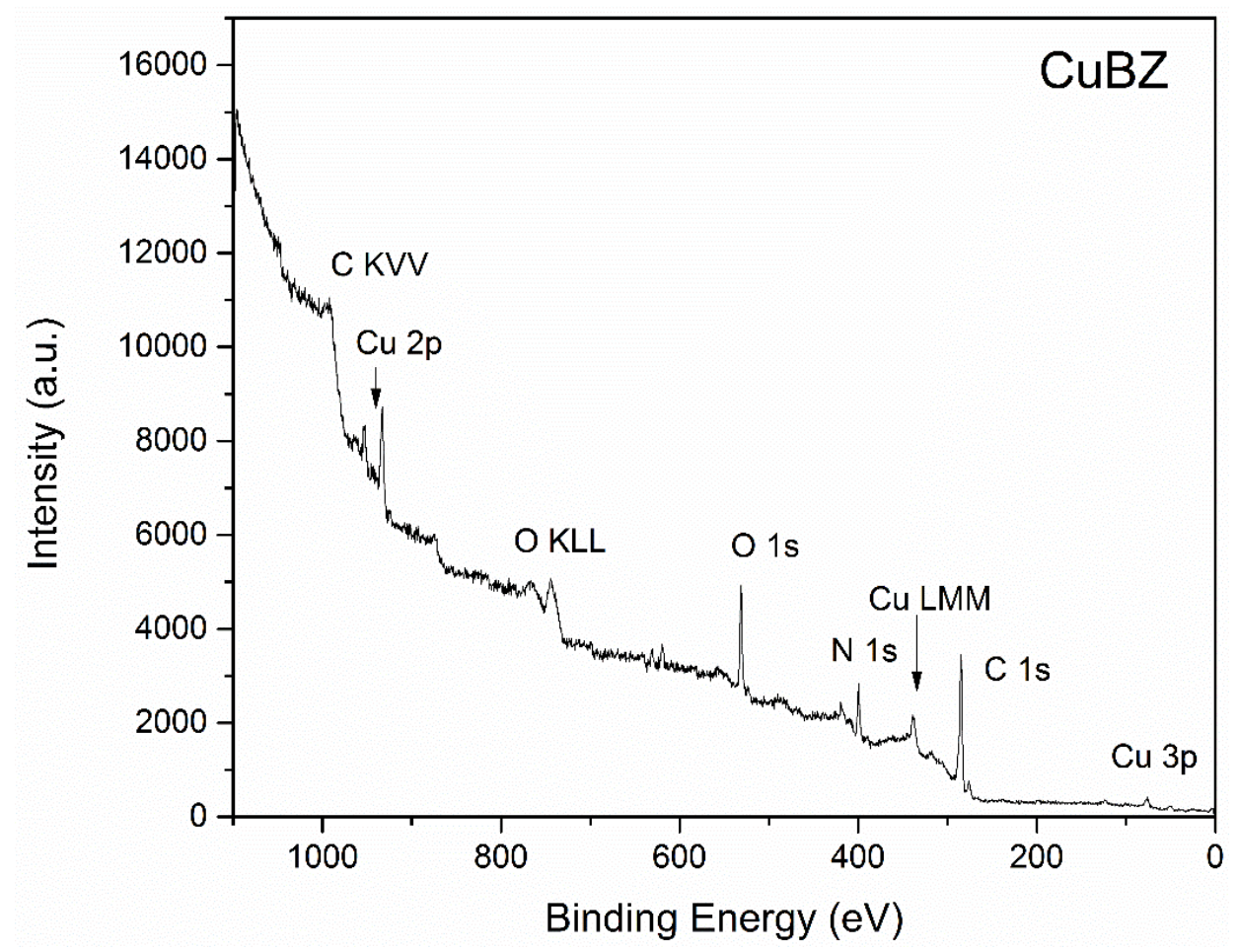

Figure S14. XPS of Cuedtabz 


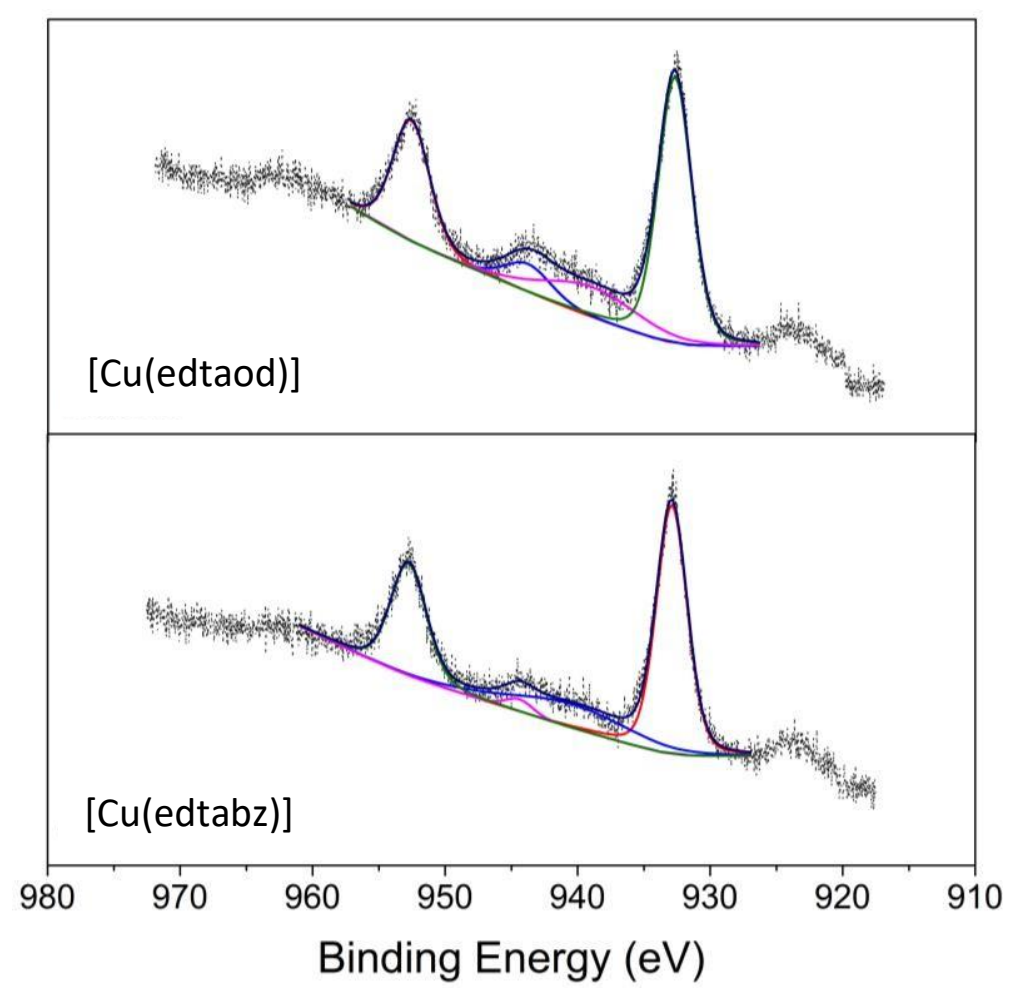

Figure S15. XPS spectra of $\mathrm{Cu} 2 \mathrm{p}$ of $\mathrm{Cu}$ complex

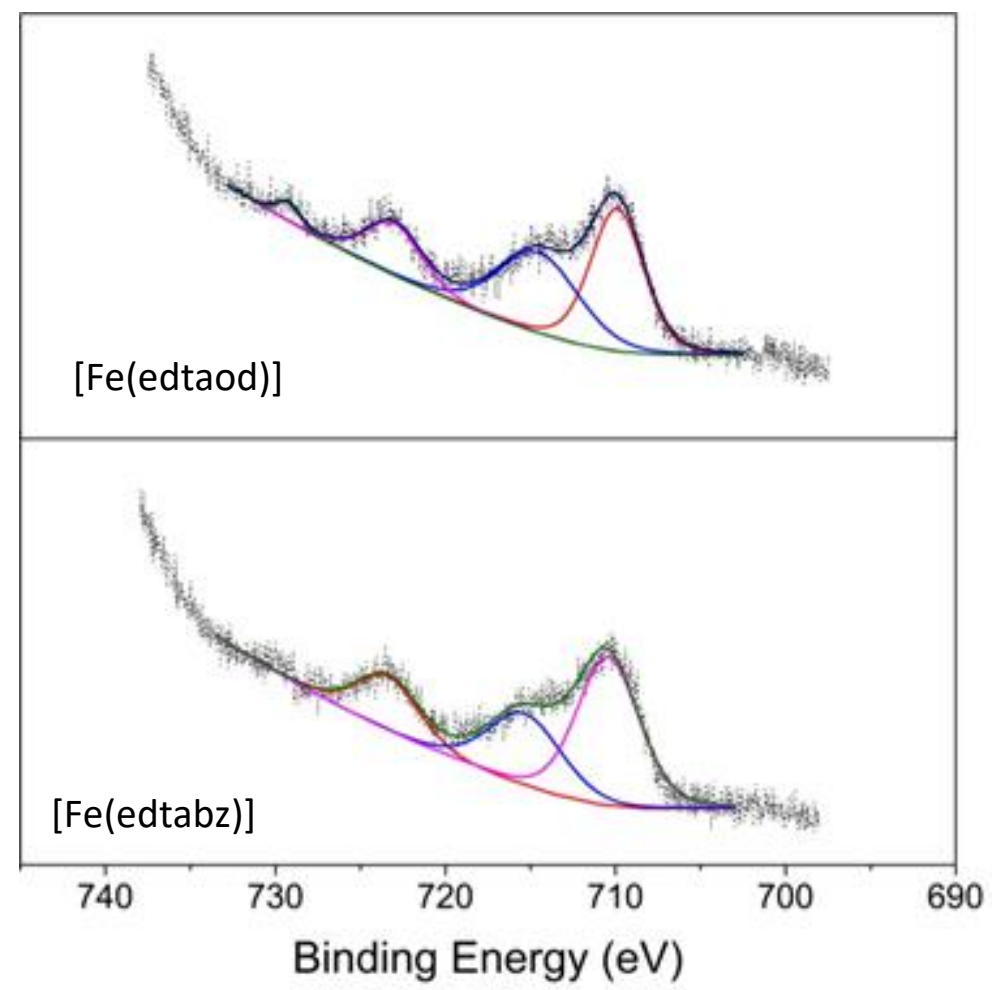

Figure S16. XPS spectra of Fe 2p of Fe complex 
Table S1. Parameters used for each titration system in ITC.

\begin{tabular}{cccccc}
\hline System & {$[\mathbf{L}] \mathbf{m M}$} & {$[\mathbf{M}] \mathbf{m M}$} & \# injections & Vol $(\boldsymbol{\mu L})$ & Spacing $(\mathbf{s})$ \\
\hline$[\mathbf{F e}($ edtabz) & 0.60 & 0.05 & 26 & 10 & 600 \\
{$[\mathbf{C u}($ edtabz) } & 0.30 & 0.025 & 36 & 6 & 300 \\
{$[\mathbf{F e}($ edtaod $)]$} & 0.60 & 0.05 & 26 & 10 & 600 \\
{$[$ Cu(edtaod)] } & 0.55 & 0.05 & 26 & 10 & 360 \\
\hline
\end{tabular}

Table S2 Fractional Atomic Coordinates for edtabzH2 $\left(\times 10^{4}\right)$ and Equivalent Isotropic Displacement Parameters $\left(\AA^{2} \times 10^{3}\right)$ for udlafunm. $U_{\text {eq }}$ is defined as $1 / 3$ of of the trace of the orthogonalised $\mathrm{U}_{\mathrm{IJ}}$ tensor.

\begin{tabular}{|c|c|c|c|c|}
\hline Atom & $x$ & $y$ & $z$ & $\mathrm{U}(\mathbf{e q})$ \\
\hline N1 & $6050(5)$ & $385(3)$ & $3288.7(16)$ & $36.5(10)$ \\
\hline $\mathrm{N} 2$ & $4299(5)$ & $1417(3)$ & $2383.9(16)$ & $33.2(9)$ \\
\hline N3 & $2717(6)$ & $270(3)$ & 4375.7(17) & $47.5(11)$ \\
\hline N4 & $455(5)$ & $2800(3)$ & $2950.0(19)$ & $42.1(11)$ \\
\hline $\mathrm{O} 1$ & $3182(7)$ & $1471(3)$ & $3745.8(18)$ & $81.9(15)$ \\
\hline $\mathrm{O} 2$ & $3505(4)$ & $3097(3)$ & $2900.9(15)$ & $43.0(9)$ \\
\hline $\mathrm{O} 3$ & $7926(5)$ & $-1441(3)$ & $3502.7(16)$ & $48.2(9)$ \\
\hline $\mathrm{O} 4$ & $10528(4)$ & $-780(3)$ & $3242.1(18)$ & $53.5(11)$ \\
\hline O5 & $7763(5)$ & 1901(3) & $2317.6(16)$ & $56.6(11)$ \\
\hline O6 & $7584(5)$ & $2662(3)$ & $1499.2(16)$ & $56.0(11)$ \\
\hline $\mathrm{C} 1$ & $3680(8)$ & $742(4)$ & $3982(2)$ & $50.8(15)$ \\
\hline $\mathrm{C} 2$ & $5527(8)$ & $282(5)$ & $3872(2)$ & $54.8(15)$ \\
\hline $\mathrm{C} 3$ & $4846(6)$ & $-139(3)$ & $2907(2)$ & $36.7(11)$ \\
\hline $\mathrm{C} 4$ & $4734(7)$ & $345(3)$ & $2345(2)$ & $36.6(11)$ \\
\hline $\mathrm{C} 5$ & $2355(6)$ & $1618(3)$ & $2512(2)$ & $35.6(11)$ \\
\hline C6 & $2170(6)$ & 2601(4) & $2805.6(19)$ & $33.8(11)$ \\
\hline $\mathrm{C} 7$ & 7984(7) & $242(4)$ & $3194(2)$ & 41.1(12) \\
\hline $\mathrm{C} 8$ & $8774(7)$ & $-752(4)$ & $3334(2)$ & $38.0(12)$ \\
\hline C9 & $4913(6)$ & $1947(4)$ & $1871(2)$ & $38.0(12)$ \\
\hline $\mathrm{C} 10$ & $6952(7)$ & $2179(4)$ & $1900(2)$ & $38.2(12)$ \\
\hline C11 & $1005(7)$ & $549(4)$ & $4606(2)$ & 43.1(13) \\
\hline $\mathrm{C} 12$ & 11(9) & $-154(5)$ & $4879(2)$ & $58.1(16)$ \\
\hline $\mathrm{C} 13$ & $-1594(9)$ & $83(6)$ & $5146(3)$ & $69.8(19)$ \\
\hline C14 & $-2234(10)$ & $1029(6)$ & $5130(3)$ & $73(2)$ \\
\hline $\mathrm{C} 15$ & $-1245(10)$ & $1729(5)$ & $4850(3)$ & $68.0(19)$ \\
\hline $\mathrm{C} 16$ & $348(8)$ & $1493(5)$ & $4581(2)$ & $56.8(16)$ \\
\hline $\mathrm{C} 17$ & $-231(7)$ & $3555(4)$ & $3298(2)$ & $38.3(12)$ \\
\hline $\mathrm{C} 18$ & $-1929(8)$ & $3423(4)$ & $3528(2)$ & $54.6(15)$ \\
\hline C19 & $-2679(10)$ & $4131(5)$ & $3867(3)$ & $66.2(18)$ \\
\hline $\mathrm{C} 20$ & $-1761(10)$ & $4979(5)$ & $3970(2)$ & $63.7(19)$ \\
\hline $\mathrm{C} 21$ & $-93(10)$ & $5112(5)$ & $3732(2)$ & $64.5(18)$ \\
\hline $\mathrm{C} 22$ & 697(9) & $4411(4)$ & $3404(2)$ & $54.5(15)$ \\
\hline $\mathrm{O} 31$ & $1174(7)$ & 1811(3) & 9536.1(18) & $70.7(13)$ \\
\hline $\mathrm{O} 32$ & $7686(8)$ & $2545(4)$ & $9504(2)$ & $88.2(16)$ \\
\hline
\end{tabular}




\begin{tabular}{|c|c|c|c|c|c|c|}
\hline \multicolumn{7}{|c|}{ 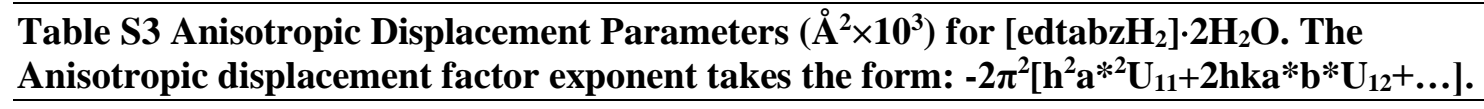 } \\
\hline Atom & $\mathbf{U}_{11}$ & $\mathbf{U}_{22}$ & $\mathbf{U}_{33}$ & $\mathbf{U}_{23}$ & $\mathrm{U}_{13}$ & $\mathrm{U}_{12}$ \\
\hline$\overline{\mathrm{N} 1}$ & $32(2)$ & $42(2)$ & $36(2)$ & $4(2)$ & $-0.4(18)$ & $-1.5(19)$ \\
\hline $\mathrm{N} 2$ & $22(2)$ & $37(2)$ & $41(2)$ & $-2(2)$ & $-3.8(17)$ & $0.0(17)$ \\
\hline N3 & $51(3)$ & $52(3)$ & $40(2)$ & $3(2)$ & $-2(2)$ & $13(2)$ \\
\hline N4 & $26(2)$ & $45(3)$ & $55(3)$ & $-4(2)$ & $-4(2)$ & $-1.8(19)$ \\
\hline $\mathrm{O} 1$ & 103(4) & $76(3)$ & $68(3)$ & $29(3)$ & $31(3)$ & $44(3)$ \\
\hline $\mathrm{O} 2$ & $32.0(19)$ & $46(2)$ & $51(2)$ & $-3.6(18)$ & $3.5(17)$ & $-6.3(16)$ \\
\hline $\mathrm{O} 3$ & $33.6(19)$ & $44(2)$ & $67(2)$ & 12.2(19) & $-1.0(19)$ & $-2.2(18)$ \\
\hline $\mathrm{O} 4$ & $27(2)$ & $49(2)$ & $85(3)$ & $2(2)$ & 10.1(19) & $-2.0(17)$ \\
\hline $\mathrm{O} 5$ & $33(2)$ & $73(3)$ & $64(3)$ & $14(2)$ & $-14.6(19)$ & $-20.5(19)$ \\
\hline O6 & $44(2)$ & $70(3)$ & $54(2)$ & $10(2)$ & $2.8(19)$ & $-18(2)$ \\
\hline $\mathrm{C} 1$ & $65(4)$ & $51(4)$ & $36(3)$ & $4(3)$ & $2(3)$ & $17(3)$ \\
\hline $\mathrm{C} 2$ & $57(4)$ & $59(4)$ & $49(3)$ & $8(3)$ & $3(3)$ & $10(3)$ \\
\hline $\mathrm{C} 3$ & $29(2)$ & $30(3)$ & $51(3)$ & $7(2)$ & $2(2)$ & $0(2)$ \\
\hline $\mathrm{C} 4$ & $29(2)$ & $34(3)$ & $47(3)$ & $-11(2)$ & $-3(2)$ & $-1(2)$ \\
\hline $\mathrm{C} 5$ & $24(2)$ & $39(3)$ & $45(3)$ & $5(2)$ & $-2(2)$ & $-5(2)$ \\
\hline C6 & $27(2)$ & $40(3)$ & $34(3)$ & $6(2)$ & $-6(2)$ & $-4(2)$ \\
\hline $\mathrm{C} 7$ & $33(3)$ & $46(3)$ & $44(3)$ & $5(2)$ & $-3(2)$ & $-10(3)$ \\
\hline $\mathrm{C} 8$ & $28(3)$ & $50(3)$ & $37(3)$ & $-2(2)$ & $-5(2)$ & $-14(2)$ \\
\hline C9 & $33(3)$ & $37(3)$ & $44(3)$ & $4(2)$ & $-6(2)$ & $0(2)$ \\
\hline $\mathrm{C} 10$ & $36(3)$ & $37(3)$ & $41(3)$ & $-5(2)$ & $1(2)$ & $1(2)$ \\
\hline $\mathrm{C} 11$ & $46(3)$ & $51(3)$ & $32(3)$ & $-14(3)$ & $-3(2)$ & $3(3)$ \\
\hline $\mathrm{C} 12$ & $63(4)$ & $57(4)$ & $54(3)$ & $-2(3)$ & $2(3)$ & $3(3)$ \\
\hline $\mathrm{C} 13$ & $64(5)$ & $84(5)$ & $62(4)$ & $-1(4)$ & $8(3)$ & $-7(4)$ \\
\hline $\mathrm{C} 14$ & $52(4)$ & $93(5)$ & $74(4)$ & $-18(4)$ & $10(4)$ & $10(4)$ \\
\hline $\mathrm{C} 15$ & $68(4)$ & $65(5)$ & $71(4)$ & $-13(4)$ & $2(4)$ & $15(4)$ \\
\hline $\mathrm{C} 16$ & $62(4)$ & $58(4)$ & $51(3)$ & $-16(3)$ & $5(3)$ & $2(3)$ \\
\hline $\mathrm{C} 17$ & $37(3)$ & $42(3)$ & $36(3)$ & $3(2)$ & $-1(2)$ & $13(2)$ \\
\hline $\mathrm{C} 18$ & $50(4)$ & $52(4)$ & $62(4)$ & $2(3)$ & $1(3)$ & $9(3)$ \\
\hline C19 & $62(4)$ & $75(5)$ & $62(4)$ & $9(4)$ & $14(3)$ & $15(4)$ \\
\hline C20 & $80(5)$ & $66(5)$ & $45(4)$ & $0(3)$ & $8(3)$ & $31(4)$ \\
\hline $\mathrm{C} 21$ & $87(5)$ & $56(4)$ & $51(4)$ & $-9(3)$ & $-7(4)$ & $5(4)$ \\
\hline $\mathrm{C} 22$ & $60(4)$ & $46(3)$ & $58(4)$ & $-5(3)$ & $7(3)$ & $-3(3)$ \\
\hline $\mathrm{O} 31$ & $83(3)$ & $70(3)$ & $59(3)$ & $-5(2)$ & $-5(3)$ & 1(3) \\
\hline $\mathrm{O} 32$ & $91(4)$ & $98(4)$ & $75(3)$ & $-36(3)$ & $-2(3)$ & $12(3)$ \\
\hline
\end{tabular}

Table S4 Bond Lengths for [edtabzH $\mathrm{H}_{2} \cdot 2 \mathrm{H}_{2} \mathrm{O}$.

\begin{tabular}{|c|c|c|c|}
\hline Atom Atom & Length/Å & Atom Atom & Length/Å \\
\hline $\mathrm{N} 1 \quad \mathrm{C} 2$ & $1.449(6)$ & $\mathrm{C} 3$ & $1.496(7)$ \\
\hline $\mathrm{C} 3$ & $1.455(6)$ & $\mathrm{C} 5$ & $1.517(7)$ \\
\hline $\mathrm{N} 1 \quad \mathrm{C} 7$ & $1.448(6)$ & $\mathrm{C} 7$ & $1.511(7)$ \\
\hline
\end{tabular}




\begin{tabular}{llllll}
$\mathrm{N} 2$ & $\mathrm{C} 4$ & $1.499(6)$ & $\mathrm{C} 9$ & $\mathrm{C} 10$ & $1.529(7)$ \\
$\mathrm{N} 2$ & $\mathrm{C} 5$ & $1.482(6)$ & $\mathrm{C} 11$ & $\mathrm{C} 12$ & $1.369(8)$ \\
$\mathrm{N} 2$ & $\mathrm{C} 9$ & $1.490(6)$ & $\mathrm{C} 11$ & $\mathrm{C} 16$ & $1.375(8)$ \\
$\mathrm{N} 3$ & $\mathrm{C} 1$ & $1.340(7)$ & $\mathrm{C} 12$ & $\mathrm{C} 13$ & $1.375(9)$ \\
$\mathrm{N} 3$ & $\mathrm{C} 11$ & $1.421(7)$ & $\mathrm{C} 13$ & $\mathrm{C} 14$ & $1.372(9)$ \\
$\mathrm{N} 4$ & $\mathrm{C} 6$ & $1.331(6)$ & $\mathrm{C} 14$ & $\mathrm{C} 15$ & $1.371(9)$ \\
$\mathrm{N} 4$ & $\mathrm{C} 17$ & $1.416(6)$ & $\mathrm{C} 15$ & $\mathrm{C} 16$ & $1.370(9)$ \\
$\mathrm{O} 1$ & $\mathrm{C} 1$ & $1.198(6)$ & $\mathrm{C} 17$ & $\mathrm{C} 18$ & $1.371(7)$ \\
$\mathrm{O} 2$ & $\mathrm{C} 6$ & $1.212(5)$ & $\mathrm{C} 17$ & $\mathrm{C} 22$ & $1.374(8)$ \\
$\mathrm{O} 3$ & $\mathrm{C} 8$ & $1.196(6)$ & $\mathrm{C} 18$ & $\mathrm{C} 19$ & $1.374(8)$ \\
$\mathrm{O} 4$ & $\mathrm{C} 8$ & $1.305(6)$ & $\mathrm{C} 19$ & $\mathrm{C} 20$ & $1.360(9)$ \\
$\mathrm{O} 5$ & $\mathrm{C} 10$ & $1.220(6)$ & $\mathrm{C} 20$ & $\mathrm{C} 21$ & $1.360(9)$ \\
$\mathrm{O} 6$ & $\mathrm{C} 10$ & $1.250(6)$ & $\mathrm{C} 21$ & $\mathrm{C} 22$ & $1.365(8)$ \\
$\mathrm{C} 1$ & $\mathrm{C} 2$ & $1.514(8)$ & & & \\
\hline
\end{tabular}

\begin{tabular}{|c|c|c|c|c|c|c|c|}
\hline \multicolumn{8}{|c|}{ Table S5 Bond Angles for [edtabzH 2$] \cdot 2 \mathrm{H}_{2} \mathrm{O}$. } \\
\hline \multicolumn{3}{|c|}{ Atom Atom Atom } & \multirow{2}{*}{$\begin{array}{r}\text { Angle }^{\circ} \\
113.1(4)\end{array}$} & \multicolumn{3}{|c|}{ Atom Atom Atom } & \multirow{2}{*}{$\frac{\text { Angle }^{\circ}}{111.5(4)}$} \\
\hline $\mathrm{C} 2$ & $\mathrm{~N} 1$ & $\mathrm{C} 3$ & & $\mathrm{O} 4$ & $\mathrm{C} 8$ & $\mathrm{C} 7$ & \\
\hline $\mathrm{C} 7$ & $\mathrm{~N} 1$ & $\mathrm{C} 2$ & $113.3(4)$ & $\mathrm{N} 2$ & $\mathrm{C} 9$ & $\mathrm{C} 10$ & $111.0(4)$ \\
\hline $\mathrm{C} 7$ & $\mathrm{~N} 1$ & $\mathrm{C} 3$ & $115.4(4)$ & O5 & $\mathrm{C} 10$ & O6 & $127.4(5)$ \\
\hline $\mathrm{C} 5$ & $\mathrm{~N} 2$ & $\mathrm{C} 4$ & $113.4(4)$ & O5 & $\mathrm{C} 10$ & $\mathrm{C} 9$ & $116.7(5)$ \\
\hline $\mathrm{C} 5$ & $\mathrm{~N} 2$ & $\mathrm{C} 9$ & $111.7(4)$ & O6 & $\mathrm{C} 10$ & $\mathrm{C} 9$ & $115.9(5)$ \\
\hline C9 & $\mathrm{N} 2$ & $\mathrm{C} 4$ & $111.0(4)$ & $\mathrm{C} 12$ & $\mathrm{C} 11$ & N3 & $117.8(5)$ \\
\hline $\mathrm{C} 1$ & N3 & $\mathrm{C} 11$ & $127.4(5)$ & $\mathrm{C} 12$ & $\mathrm{C} 11$ & $\mathrm{C} 16$ & $119.3(5)$ \\
\hline C6 & $\mathrm{N} 4$ & $\mathrm{C} 17$ & $129.5(4)$ & $\mathrm{C} 16$ & $\mathrm{C} 11$ & N3 & $122.9(5)$ \\
\hline N3 & $\mathrm{C} 1$ & $\mathrm{C} 2$ & $113.2(5)$ & $\mathrm{C} 11$ & $\mathrm{C} 12$ & $\mathrm{C} 13$ & $120.7(6)$ \\
\hline $\mathrm{O} 1$ & $\mathrm{C} 1$ & N3 & $124.5(5)$ & $\mathrm{C} 14$ & $\mathrm{C} 13$ & $\mathrm{C} 12$ & $120.0(7)$ \\
\hline $\mathrm{O} 1$ & $\mathrm{C} 1$ & $\mathrm{C} 2$ & $122.3(6)$ & $\mathrm{C} 15$ & $\mathrm{C} 14$ & $\mathrm{C} 13$ & $119.1(6)$ \\
\hline $\mathrm{N} 1$ & $\mathrm{C} 2$ & $\mathrm{C} 1$ & $111.3(5)$ & $\mathrm{C} 16$ & $\mathrm{C} 15$ & $\mathrm{C} 14$ & $121.0(6)$ \\
\hline N1 & $\mathrm{C} 3$ & $\mathrm{C} 4$ & $112.2(4)$ & $\mathrm{C} 15$ & $\mathrm{C} 16$ & C11 & $119.8(6)$ \\
\hline C3 & $\mathrm{C} 4$ & $\mathrm{~N} 2$ & $112.7(4)$ & $\mathrm{C} 18$ & $\mathrm{C} 17$ & N4 & $117.5(5)$ \\
\hline $\mathrm{N} 2$ & $\mathrm{C} 5$ & C6 & $110.1(4)$ & $\mathrm{C} 18$ & $\mathrm{C} 17$ & $\mathrm{C} 22$ & $119.2(5)$ \\
\hline N4 & C6 & $\mathrm{C} 5$ & $112.6(4)$ & $\mathrm{C} 22$ & $\mathrm{C} 17$ & N4 & $123.3(5)$ \\
\hline $\mathrm{O} 2$ & C6 & N4 & $126.8(5)$ & $\mathrm{C} 17$ & $\mathrm{C} 18$ & C19 & $120.3(6)$ \\
\hline $\mathrm{O} 2$ & C6 & $\mathrm{C} 5$ & $120.5(4)$ & $\mathrm{C} 20$ & C19 & $\mathrm{C} 18$ & $120.4(6)$ \\
\hline $\mathrm{N} 1$ & $\mathrm{C} 7$ & $\mathrm{C} 8$ & $117.5(4)$ & C19 & $\mathrm{C} 20$ & $\mathrm{C} 21$ & $118.8(6)$ \\
\hline $\mathrm{O} 3$ & $\mathrm{C} 8$ & $\mathrm{O} 4$ & $123.1(5)$ & $\mathrm{C} 20$ & $\mathrm{C} 21$ & $\mathrm{C} 22$ & $121.8(6)$ \\
\hline $\mathrm{O} 3$ & $\mathrm{C} 8$ & $\mathrm{C} 7$ & $125.4(4)$ & $\mathrm{C} 21$ & $\mathrm{C} 22$ & $\mathrm{C} 17$ & $119.4(6)$ \\
\hline
\end{tabular}


Table S6 Torsion Angles for [edtabzH $\left.\mathrm{e}_{2}\right] \cdot 2 \mathrm{H}_{2} \mathrm{O}$.

\begin{tabular}{|c|c|c|c|c|}
\hline $\begin{array}{ll}\mathbf{A} & \mathbf{B} \\
\end{array}$ & $\mathbf{C}$ & Angle $/^{\circ}$ & $\begin{array}{llll}\mathbf{A} & \mathbf{B} & \mathbf{C} & \mathbf{D}\end{array}$ & Angle $/^{\circ}$ \\
\hline $\mathrm{N} 1 \mathrm{C} 3$ & $\mathrm{C} 4 \quad \mathrm{~N} 2$ & $-51.3(5)$ & C6 N4 C17C18 & $-159.1(5)$ \\
\hline N1 C7 & $\mathrm{C} 8 \mathrm{O} 3$ & $2.3(8)$ & C6 N4 C17C22 & $22.4(8)$ \\
\hline $\mathrm{N} 1 \mathrm{C} 7$ & C8 $\mathrm{O} 4$ & $-178.6(4)$ & C7 N1 C2 C1 & $162.0(5)$ \\
\hline $\mathrm{N} 2 \mathrm{C} 5$ & C6 N4 & $-177.5(4)$ & C7 $\quad$ N1 $\mathrm{C} 3 \quad \mathrm{C} 4$ & $-76.9(5)$ \\
\hline $\mathrm{N} 2 \mathrm{C} 5$ & C6 $\mathrm{O} 2$ & $0.3(6)$ & $\begin{array}{llll}\text { C9 } & \text { N2 } & \text { C4 } & \text { C3 }\end{array}$ & $157.3(4)$ \\
\hline $\mathrm{N} 2 \mathrm{C} 9$ & $\mathrm{C} 10 \mathrm{O} 5$ & $0.7(7)$ & C9 N2 C5 C6 & $-80.2(5)$ \\
\hline $\mathrm{N} 2 \mathrm{C} 9$ & C10O6 & $-177.0(4)$ & C11 N3 C1 O1 & $-3.0(10)$ \\
\hline $\mathrm{N} 3 \mathrm{C} 1$ & $\mathrm{C} 2 \mathrm{~N} 1$ & $146.7(5)$ & $\mathrm{C} 11 \mathrm{~N} 3 \mathrm{C} 1 \mathrm{C} 2$ & $175.0(5)$ \\
\hline $\mathrm{N} 3 \mathrm{C} 1$ & $1 \mathrm{C} 12 \mathrm{C} 13$ & $175.3(5)$ & C11 C12 C13 C14 & $1.4(10)$ \\
\hline $\mathrm{N} 3 \mathrm{C} 1$ & $1 \mathrm{C} 16 \mathrm{C} 15$ & $-174.8(5)$ & C12C11 C16C15 & $2.9(8)$ \\
\hline $\mathrm{N} 4 \mathrm{C} 1$ & 7 C18C19 & $-179.6(5)$ & C12 C13 C14 C15 & $-0.6(11)$ \\
\hline $\mathrm{N} 4 \mathrm{C} 1$ & 7 C22C21 & $178.1(5)$ & C13 C14 C15 C16 & $1.0(11)$ \\
\hline $\mathrm{O} 1 \mathrm{C} 1$ & C2 N1 & $-35.2(8)$ & C14 C15 C16C11 & $-2.2(10)$ \\
\hline C1 N3 & C11 C12 & $161.7(6)$ & C16 C11 C12C13 & $-2.5(9)$ \\
\hline C1 N3 & C11 C16 & $-20.5(8)$ & $\mathrm{C} 17 \mathrm{~N} 4 \mathrm{C} 6 \mathrm{O} 2$ & $-8.1(9)$ \\
\hline C2 N1 & C3 C4 & $150.3(4)$ & C17N4 C6 C5 & $169.5(4)$ \\
\hline $\mathrm{C} 2 \mathrm{~N} 1$ & $\mathrm{C} 7 \mathrm{C} 8$ & $62.1(6)$ & C17 C18 C19 C20 & $1.2(9)$ \\
\hline C3 N1 & $\mathrm{C} 2 \mathrm{C} 1$ & $-64.2(6)$ & C18 C17 C22 C21 & $-0.4(8)$ \\
\hline C3 N1 & C7 C8 & $-70.5(6)$ & C18 C19 C20 C21 & $0.1(9)$ \\
\hline $\mathrm{C} 4 \mathrm{~N} 2$ & C5 C6 & $153.5(4)$ & C19C20C21 C22 & $-1.5(9)$ \\
\hline $\mathrm{C} 4 \mathrm{~N} 2$ & C9 C10 & $-81.3(5)$ & C20 C21 C22 C17 & $1.7(9)$ \\
\hline $\mathrm{C} 5 \mathrm{~N} 2$ & $\mathrm{C} 4 \mathrm{C} 3$ & $-76.0(5)$ & C22 C17 C18 C19 & $-1.0(8)$ \\
\hline C5 N2 & C9 C10 & $151.0(4)$ & & \\
\hline
\end{tabular}


Table S7. Hydrogen Atom Coordinates $\left(\AA \times 10^{4}\right)$ and Isotropic Displacement Parameters $\left(\AA^{2} \times 10^{3}\right)$ for $[$ edtabzH 2$] \cdot 2 \mathrm{H}_{2} \mathrm{O}$.

\begin{tabular}{|c|c|c|c|c|}
\hline Atom & $x$ & $y$ & $z$ & $\mathrm{U}(\mathbf{e q})$ \\
\hline$\overline{\mathrm{H} 2}$ & 5014 & 1679 & 2697 & 40 \\
\hline H3 & 3188 & -263 & 4504 & 57 \\
\hline $\mathrm{H} 4$ & -354 & 2413 & 2811 & 51 \\
\hline $\mathrm{H} 4 \mathrm{~A}$ & 10908 & -1337 & 3301 & 80 \\
\hline $\mathrm{H} 2 \mathrm{~A}$ & 6437 & 594 & 4107 & 66 \\
\hline $\mathrm{H} 2 \mathrm{~B}$ & 5485 & -408 & 3969 & 66 \\
\hline $\mathrm{H} 3 \mathrm{~A}$ & 3634 & -171 & 3070 & 44 \\
\hline H3B & 5287 & -805 & 2861 & 44 \\
\hline $\mathrm{H} 4 \mathrm{~B}$ & 3801 & 21 & 2124 & 44 \\
\hline $\mathrm{H} 4 \mathrm{C}$ & 5891 & 263 & 2153 & 44 \\
\hline H5A & 1874 & 1103 & 2750 & 43 \\
\hline H5B & 1654 & 1625 & 2167 & 43 \\
\hline H7A & 8639 & 730 & 3410 & 49 \\
\hline H7B & 8230 & 373 & 2801 & 49 \\
\hline H9A & 4230 & 2552 & 1833 & 46 \\
\hline H9B & 4670 & 1546 & 1543 & 46 \\
\hline H12 & 424 & -799 & 4885 & 70 \\
\hline H13 & -2245 & -397 & 5338 & 84 \\
\hline H14 & -3323 & 1193 & 5306 & 88 \\
\hline H15 & -1661 & 2373 & 4842 & 82 \\
\hline H16 & 983 & 1970 & 4382 & 68 \\
\hline H18 & -2577 & 2851 & 3454 & 65 \\
\hline H19 & -3822 & 4030 & 4026 & 79 \\
\hline $\mathrm{H} 20$ & -2265 & 5459 & 4200 & 76 \\
\hline $\mathrm{H} 21$ & 527 & 5697 & 3795 & 77 \\
\hline $\mathrm{H} 22$ & 1853 & 4511 & 3253 & 65 \\
\hline $\mathrm{H} 31 \mathrm{~A}$ & 1524 & 2109 & 9242 & 106 \\
\hline H31B & 1651 & 2079 & 9822 & 106 \\
\hline $\mathrm{H} 32 \mathrm{~A}$ & 8657 & 2223 & 9568 & 132 \\
\hline H32B & 7086 & 2267 & 9244 & 132 \\
\hline
\end{tabular}

Table S8 Fractional Atomic Coordinates $\left(\times 10^{4}\right)$ and Equivalent Isotropic Displacement Parameters $\left(\AA^{2} \times 1^{3}\right)$ for $\left\{[\mathrm{Fe}(\text { edtaod })]_{2} \mathrm{O}\right\} \cdot 4.4 \mathrm{H}_{2} \mathrm{O}$. $\mathrm{U}_{\text {eq }}$ is defined as $1 / 3$ of of the trace of the orthogonalised $\mathrm{U}_{\mathrm{IJ}}$ tensor.

\begin{tabular}{lllll}
\hline Atom & $\boldsymbol{x}$ & $\boldsymbol{y}$ & $\boldsymbol{z}$ & $\mathbf{U}(\mathbf{e q})$ \\
\hline $\mathrm{Fe} 2$ & $2914.8(2)$ & $3191.9(2)$ & $4319.4(2)$ & $10.19(6)$ \\
$\mathrm{Fe} 1$ & $4227.8(2)$ & $3410.9(2)$ & $3222.8(2)$ & $8.91(6)$ \\
$\mathrm{O} 1 \mathrm{~B}$ & $3302.6(6)$ & $2131.5(7)$ & $4305.9(5)$ & $12.2(2)$ \\
\hline
\end{tabular}




\begin{tabular}{|c|c|c|c|c|}
\hline $\mathrm{O} 1 \mathrm{~A}$ & $4065.8(7)$ & $2303.6(6)$ & $3194.7(5)$ & $12.9(2)$ \\
\hline $\mathrm{O} 4 \mathrm{~A}$ & $4519.2(7)$ & $4447.6(6)$ & $2996.0(5)$ & $13.0(2)$ \\
\hline $\mathrm{O} 2 \mathrm{~A}$ & $5160.8(7)$ & $3276.1(7)$ & $3682.5(5)$ & $14.1(2)$ \\
\hline O6A & 2899.4(7) & $2046.2(7)$ & $1940.4(5)$ & $14.8(2)$ \\
\hline $\mathrm{O} 4 \mathrm{~B}$ & $2446.6(7)$ & $4128.9(7)$ & $4631.3(5)$ & $14.7(2)$ \\
\hline O009 & $3526.7(7)$ & $3548.0(7)$ & $3768.2(5)$ & $13.5(2)$ \\
\hline $\mathrm{O} 2 \mathrm{~B}$ & 2007.2(7) & $2919.8(7)$ & $3853.3(5)$ & $15.0(2)$ \\
\hline O6B & $4405.6(7)$ & $1913.9(7)$ & $5506.1(5)$ & $16.2(3)$ \\
\hline O5A & $4229.4(7)$ & $5457.1(7)$ & $2477.8(6)$ & $17.6(3)$ \\
\hline O5B & $2586.6(8)$ & $5074.0(7)$ & $5252.1(5)$ & $20.8(3)$ \\
\hline $\mathrm{O} 3 \mathrm{~W}$ & $1906.3(8)$ & $914.2(8)$ & $1687.5(6)$ & 21.1(3) \\
\hline $\mathrm{O} 2 \mathrm{~W}$ & $3017.9(8)$ & $-149.6(9)$ & $1467.1(6)$ & $26.6(3)$ \\
\hline $\mathrm{O} 3 \mathrm{~A}$ & $6387.3(7)$ & $3130.4(9)$ & $3672.2(6)$ & $27.1(3)$ \\
\hline $\mathrm{O} 1 \mathrm{~W}$ & $4386.8(8)$ & 498.7(8) & $1651.9(6)$ & $23.6(3)$ \\
\hline $\mathrm{O} 3 \mathrm{~B}$ & $930.0(7)$ & $2304.5(8)$ & $3864.0(6)$ & $24.8(3)$ \\
\hline $\mathrm{N} 4 \mathrm{~A}$ & $2618.0(8)$ & 2103.2(8) & $2905.2(6)$ & $12.7(3)$ \\
\hline N4B & $4723.4(8)$ & $2067.6(8)$ & $4554.7(6)$ & $11.8(3)$ \\
\hline N1B & $3452.9(8)$ & $1033.2(8)$ & $4765.2(6)$ & $11.5(3)$ \\
\hline $\mathrm{N} 3 \mathrm{~A}$ & $3564.7(7)$ & $3562.6(8)$ & $2386.5(6)$ & $9.9(3)$ \\
\hline N3B & $3585.7(8)$ & $3379.8(8)$ & $5122.4(6)$ & $13.2(3)$ \\
\hline N1A & $4017.6(8)$ & $1295.6(8)$ & $2618.7(6)$ & $13.5(3)$ \\
\hline $\mathrm{N} 2 \mathrm{~A}$ & $5054.6(8)$ & $3015.4(8)$ & $2521.8(6)$ & $12.0(3)$ \\
\hline $\mathrm{N} 2 \mathrm{~B}$ & $2203.6(8)$ & $2600.0(8)$ & 4996.4(6) & $13.8(3)$ \\
\hline $\mathrm{C} 1 \mathrm{~B}$ & 3091.7(9) & $1657.9(9)$ & $4668.6(7)$ & 11.7(3) \\
\hline $\mathrm{C} 8 \mathrm{~B}$ & $2808.3(10)$ & $4498.9(10)$ & $5013.4(7)$ & $16.0(3)$ \\
\hline C7A & $3487.1(9)$ & 4381.3(9) & $2343.4(7)$ & $12.0(3)$ \\
\hline C11B & $4738.9(9)$ & $1302.7(9)$ & $4412.3(7)$ & $11.8(3)$ \\
\hline C10B & $4504.5(9)$ & $2320.0(9)$ & $5083.2(7)$ & $12.0(3)$ \\
\hline C10A & 2773.5(9) & 2411.5(9) & $2385.1(7)$ & 11.3(3) \\
\hline $\mathrm{C} 1 \mathrm{~A}$ & $4321.9(9)$ & 1925.1(9) & 2781.5(7) & 12.1(3) \\
\hline $\mathrm{C} 12 \mathrm{~B}$ & $4148.8(9)$ & $818.0(10)$ & $4525.1(7)$ & $11.8(3)$ \\
\hline C6A & 4009.1(9) & $3285.2(9)$ & $1876.6(7)$ & $12.4(3)$ \\
\hline $\mathrm{C} 8 \mathrm{~A}$ & $4129.2(9)$ & $4806.2(9)$ & $2614.6(7)$ & $12.3(3)$ \\
\hline $\mathrm{C} 12 \mathrm{~A}$ & $3399.8(10)$ & $969.9(9)$ & 2913.9(7) & 13.3(3) \\
\hline $\mathrm{C} 4 \mathrm{~A}$ & $5798.9(10)$ & $3204.5(10)$ & $3419.3(8)$ & $15.7(3)$ \\
\hline C16A & $2192.6(10)$ & $979.8(10)$ & $3364.7(7)$ & $16.3(3)$ \\
\hline $\mathrm{C} 11 \mathrm{~A}$ & $2741.9(10)$ & $1345.8(9)$ & $3046.0(7)$ & 13.1(3) \\
\hline $\mathrm{C} 16 \mathrm{~B}$ & 5381.2(9) & 1021.1(10) & $4156.2(7)$ & $14.6(3)$ \\
\hline $\mathrm{C} 2 \mathrm{~B}$ & $2386.2(10)$ & $1800.0(10)$ & $5015.9(8)$ & $16.7(3)$ \\
\hline C9A & $2790.0(9)$ & $3255.2(9)$ & $2370.9(7)$ & $11.5(3)$ \\
\hline $\mathrm{C} 13 \mathrm{~A}$ & $3478.3(11)$ & $229.8(10)$ & $3078.1(7)$ & $16.6(3)$ \\
\hline $\mathrm{C} 14 \mathrm{~B}$ & $4897.3(10)$ & $-216.7(10)$ & $4206.7(7)$ & $16.5(3)$ \\
\hline
\end{tabular}




\begin{tabular}{lllll}
\hline C5A & $4835.9(9)$ & $3387.7(9)$ & $1968.1(7)$ & $12.7(3)$ \\
C13B & $4243.3(10)$ & $58.1(10)$ & $4431.4(7)$ & $13.8(3)$ \\
C9B & $4387.4(9)$ & $3154.9(10)$ & $5124.5(8)$ & $14.3(3)$ \\
C2A & $5011.9(10)$ & $2196.1(9)$ & $2470.3(8)$ & $14.9(3)$ \\
C5B & $2361.5(10)$ & $2983.2(10)$ & $5558.8(7)$ & $16.2(3)$ \\
C6B & $3196.4(10)$ & $3053.0(11)$ & $5641.1(7)$ & $17.3(4)$ \\
C15A & $2287.2(11)$ & $252.6(10)$ & $3543.7(8)$ & $19.0(4)$ \\
C15B & $5462.6(10)$ & $268.3(10)$ & $4054.1(8)$ & $17.4(4)$ \\
C4B & $1436.7(9)$ & $2622.3(10)$ & $4114.9(8)$ & $16.7(3)$ \\
C3A & $5778.3(9)$ & $3259.0(10)$ & $2749.6(8)$ & $15.7(3)$ \\
C3B & $1439.3(9)$ & $2710.6(11)$ & $4781.8(8)$ & $17.2(4)$ \\
C14A & $2929.6(11)$ & $-127.2(10)$ & $3394.5(8)$ & $19.4(4)$ \\
C7B & $3574.4(10)$ & $4200.3(10)$ & $5160.3(8)$ & $16.7(3)$ \\
O5W & $4262.4(13)$ & $5702.9(14)$ & $4362.6(11)$ & $35.3(5)$ \\
O4W & $5310.9(16)$ & $4534.2(13)$ & $4437.4(11)$ & $42.4(6)$ \\
\hline
\end{tabular}

Table S9 Anisotropic Displacement Parameters $\left(\AA^{2} \times 10^{3}\right)$ for $\left\{[\mathrm{Fe}(\text { edtaod })]_{2} \mathrm{O}\right\} \cdot 4.4 \mathrm{H}_{2} \mathrm{O}$. The Anisotropic displacement factor exponent takes the form: $2 \pi^{2}\left[h^{2} a^{* 2} U_{11}+2 h k a * b * U_{12}+\ldots\right]$.

\begin{tabular}{lllllll}
\hline Atom $\mathbf{U}_{\mathbf{1 1}}$ & $\mathbf{U}_{\mathbf{2 2}}$ & $\mathbf{U}_{\mathbf{3 3}}$ & $\mathbf{U}_{\mathbf{2 3}}$ & $\mathbf{U}_{\mathbf{1 3}}$ & $\mathbf{U}_{\mathbf{1 2}}$ \\
\hline Fe2 & $10.28(11)$ & $12.45(12)$ & $7.84(11)$ & $1.84(8)$ & $1.43(8)$ & $3.73(9)$ \\
Fe1 & $8.95(11)$ & $9.41(11)$ & $8.37(11)$ & $1.31(8)$ & $0.97(8)$ & $0.46(8)$ \\
O1B & $11.8(6)$ & $13.5(6)$ & $11.3(5)$ & $2.4(4)$ & $1.7(4)$ & $3.0(4)$ \\
O1A & $15.4(6)$ & $10.7(6)$ & $12.8(6)$ & $0.4(4)$ & $3.3(4)$ & $-1.0(4)$ \\
O4A & $13.9(6)$ & $11.4(6)$ & $13.8(6)$ & $2.3(4)$ & $-1.6(5)$ & $-2.0(4)$ \\
O2A & $11.3(6)$ & $16.3(6)$ & $14.6(6)$ & $2.6(5)$ & $-1.0(5)$ & $0.0(5)$ \\
O6A & $19.5(6)$ & $14.3(6)$ & $10.6(5)$ & $-1.1(5)$ & $0.7(5)$ & $-3.1(5)$ \\
O4B & $16.0(6)$ & $17.3(6)$ & $10.7(5)$ & $1.6(5)$ & $1.3(5)$ & $6.6(5)$ \\
O9 & $15.1(6)$ & $14.6(6)$ & $10.8(5)$ & $1.8(4)$ & $3.7(5)$ & $2.9(5)$ \\
O2B & $12.3(6)$ & $20.7(6)$ & $11.9(6)$ & $0.9(5)$ & $0.2(4)$ & $4.5(5)$ \\
O6B & $20.2(6)$ & $16.4(6)$ & $11.9(6)$ & $2.0(5)$ & $-0.2(5)$ & $2.6(5)$ \\
O5A $19.3(6)$ & $11.8(6)$ & $21.7(6)$ & $3.7(5)$ & $-2.1(5)$ & $-1.8(5)$ \\
O5B & $27.4(7)$ & $19.3(7)$ & $15.7(6)$ & $-2.7(5)$ & $-1.0(5)$ & $12.0(5)$ \\
O3W 24.1(7) & $21.1(7)$ & $18.0(6)$ & $-0.8(5)$ & $0.9(5)$ & $-9.7(5)$ \\
O2W 29.1(8) & $31.4(8)$ & $19.2(7)$ & $-1.2(6)$ & $0.9(6)$ & $1.6(6)$ \\
O3A $12.6(6)$ & $40.6(9)$ & $28.0(7)$ & $6.0(6)$ & $-4.7(6)$ & $2.7(6)$ \\
O1W 21.4(7) & $26.8(7)$ & $22.7(7)$ & $-1.7(6)$ & $-2.5(6)$ & $8.1(6)$ \\
O3B & $13.0(6)$ & $36.1(8)$ & $25.4(7)$ & $0.6(6)$ & $-3.4(5)$ & $1.1(6)$ \\
N4A $14.4(7)$ & $12.7(7)$ & $10.8(6)$ & $-0.2(5)$ & $1.4(5)$ & $-1.3(5)$ \\
N4B $10.7(7)$ & $12.4(7)$ & $12.5(7)$ & $2.3(5)$ & $0.7(5)$ & $0.9(5)$ \\
\hline
\end{tabular}




\begin{tabular}{|c|c|c|c|c|c|}
\hline N1B $10.2(6)$ & $11.8(7)$ & $12.5(6)$ & $2.6(5)$ & $1.0(5)$ & $0.4(5)$ \\
\hline $\mathrm{N} 3 \mathrm{~A} \quad 9.5(6)$ & $10.4(6)$ & $9.8(6)$ & $0.1(5)$ & $1.8(5)$ & $-0.4(5)$ \\
\hline N3B $13.3(7)$ & $15.5(7)$ & $10.7(6)$ & $1.3(5)$ & $1.5(5)$ & $6.5(6)$ \\
\hline N1A $15.7(7)$ & $12.0(7)$ & $12.7(7)$ & $-0.6(5)$ & $1.6(5)$ & $1.4(5)$ \\
\hline N2A $10.7(7)$ & $10.8(6)$ & $14.7(6)$ & $2.4(5)$ & $2.0(5)$ & $0.1(5)$ \\
\hline N2B $11.4(7)$ & $18.2(7)$ & $11.9(6)$ & $2.7(5)$ & $2.6(5)$ & $6.0(6)$ \\
\hline C1B $10.7(7)$ & $14.2(8)$ & $10.2(7)$ & $0.1(6)$ & $-0.3(6)$ & $-0.1(6)$ \\
\hline C8B 20.1(9) & 17.2(9) & $10.6(7)$ & $4.2(6)$ & $2.6(6)$ & $6.8(7)$ \\
\hline C7A $13.4(8)$ & $10.6(7)$ & $12.0(7)$ & $2.4(6)$ & $-0.9(6)$ & $1.3(6)$ \\
\hline C11B 12.3(8) & $14.3(8)$ & $8.7(7)$ & $0.9(6)$ & $-0.9(6)$ & $2.6(6)$ \\
\hline C10B 7.7(7) & $14.9(8)$ & $13.3(8)$ & $0.1(6)$ & $-2.7(6)$ & $1.4(6)$ \\
\hline C10A 7.6(7) & $14.4(8)$ & $12.1(7)$ & $0.3(6)$ & $-1.5(6)$ & $-1.2(6)$ \\
\hline C1A $12.6(8)$ & $10.8(8)$ & $12.8(7)$ & $3.1(6)$ & $-0.8(6)$ & $2.3(6)$ \\
\hline C12B 11.8(8) & $15.5(8)$ & $8.3(7)$ & $1.0(6)$ & $-1.3(6)$ & $3.4(6)$ \\
\hline C6A 13.4(8) & $14.5(8)$ & $9.1(7)$ & $-0.5(6)$ & $2.9(6)$ & $-0.1(6)$ \\
\hline C8A $12.0(8)$ & $12.7(8)$ & $12.0(7)$ & $0.6(6)$ & $2.9(6)$ & $0.3(6)$ \\
\hline C12A 16.6(8) & $12.9(8)$ & $10.4(7)$ & $-0.1(6)$ & $-1.8(6)$ & $-3.6(6)$ \\
\hline C4A $12.5(8)$ & $12.3(8)$ & $22.2(9)$ & $4.0(7)$ & $-0.3(7)$ & $0.1(6)$ \\
\hline C16A 18.1(8) & $20.4(9)$ & $10.6(7)$ & $0.4(7)$ & $-1.6(6)$ & $-6.0(7)$ \\
\hline C11A 17.1(8) & $13.2(8)$ & $9.0(7)$ & $0.3(6)$ & $-2.4(6)$ & $-3.3(6)$ \\
\hline C16B 12.6(8) & 19.3(9) & $11.9(7)$ & $-0.5(6)$ & $1.0(6)$ & $1.5(6)$ \\
\hline C2B 13.2(8) & $18.4(9)$ & $18.4(8)$ & $5.7(7)$ & $4.9(7)$ & $2.8(7)$ \\
\hline C9A $9.2(7)$ & $13.6(8)$ & $11.7(7)$ & $0.7(6)$ & $-0.4(6)$ & $-0.2(6)$ \\
\hline C13A 23.6(9) & $12.8(8)$ & $13.3(8)$ & $-0.3(6)$ & $-5.0(7)$ & $-0.7(7)$ \\
\hline C14B 22.9(9) & $14.2(8)$ & $12.3(8)$ & $-2.8(6)$ & $-2.6(7)$ & $6.3(7)$ \\
\hline C5A $13.6(8)$ & $13.4(8)$ & $11.2(7)$ & $2.0(6)$ & $4.3(6)$ & $0.5(6)$ \\
\hline C13B 16.7(8) & $13.6(8)$ & $11.1(7)$ & $0.5(6)$ & $-2.7(6)$ & $-0.2(6)$ \\
\hline C9B 11.6(8) & $15.8(8)$ & $15.5(8)$ & $0.2(6)$ & $-1.3(6)$ & $3.7(6)$ \\
\hline C2A $13.7(8)$ & $11.8(8)$ & $19.2(8)$ & $0.3(6)$ & $4.6(6)$ & $2.3(6)$ \\
\hline C5B 17.5(9) & $21.5(9)$ & $9.8(7)$ & $2.9(7)$ & $4.1(6)$ & $5.8(7)$ \\
\hline С6B 17.6(9) & $25.0(9)$ & $9.2(7)$ & $4.4(7)$ & $2.1(6)$ & $7.8(7)$ \\
\hline C15A 25.6(10) & $19.5(9)$ & $11.9(8)$ & $3.2(7)$ & $-2.7(7)$ & $-10.9(7)$ \\
\hline C15B 16.1(8) & $22.3(9)$ & $13.8(8)$ & $-3.1(7)$ & $0.8(7)$ & $7.4(7)$ \\
\hline C4B 11.2(8) & $21.6(9)$ & $17.3(8)$ & $1.6(7)$ & $1.0(6)$ & $7.8(7)$ \\
\hline C3A $8.9(8)$ & $18.0(9)$ & $20.3(9)$ & $2.9(7)$ & $2.5(6)$ & $-0.1(6)$ \\
\hline С $3 B \quad 10.2(8)$ & 25.3(9) & $16.2(8)$ & $3.3(7)$ & $3.7(6)$ & $5.9(7)$ \\
\hline C14A 30.5(10) & $12.9(8)$ & $14.7(8)$ & $2.7(7)$ & $-6.6(7)$ & $-7.0(7)$ \\
\hline C7B 19.6(9) & $16.0(8)$ & $14.4(8)$ & $-2.1(6)$ & $-1.4(7)$ & $6.1(7)$ \\
\hline O5W 35.6(13) & $33.0(13)$ & $37.3(13)$ & $0.9(10)$ & $-8.0(10)$ & $-7.5(10)$ \\
\hline O4W 64.5(18) & $31.8(13)$ & $31.0(13)$ & $-3.1(10)$ & $-4.0(12)$ & $-15.0(12)$ \\
\hline
\end{tabular}




\begin{tabular}{|c|c|}
\hline Atom Atom Length/̊ & Atom Atom Length $/ \AA$ \\
\hline $\begin{array}{lll}\mathrm{Fe} 2 & \mathrm{O} 1 \mathrm{~B} & 2.0358(12)\end{array}$ & N3B C9B 1.500(2) \\
\hline $\mathrm{Fe} 2 \quad \mathrm{O} 4 \mathrm{~B} \quad 2.0182(12)$ & N3B C6B 1.498(2) \\
\hline $\mathrm{Fe} 2 \quad \mathrm{O} 9 \quad 1.7930(12)$ & N3B C7B 1.482(2) \\
\hline $\mathrm{Fe} 2 \quad \mathrm{O} 2 \mathrm{~B} \quad 2.0125(12)$ & N1A C1A $1.314(2)$ \\
\hline $\mathrm{Fe} 2 \quad \mathrm{~N} 3 \mathrm{~B} \quad 2.2238(15)$ & N1A C12A 1.428(2) \\
\hline $\mathrm{Fe} 2 \quad \mathrm{~N} 2 \mathrm{~B} \quad 2.2750(15)$ & N2A C5A 1.486(2) \\
\hline Fe1 O1A 2.0184(12) & N2A C2A $1.484(2)$ \\
\hline Fe1 O4A $2.0094(12)$ & N2A C3A $1.471(2)$ \\
\hline $\mathrm{Fe} 1 \quad \mathrm{O} 2 \mathrm{~A} \quad 1.9974(12)$ & N2B C2B $1.480(2)$ \\
\hline Fe1 O9 1.7919(12) & N2B C5B $1.487(2)$ \\
\hline Fe1 N3A $2.2707(14)$ & N2B C3B $1.476(2)$ \\
\hline $\mathrm{Fe} 1 \quad \mathrm{~N} 2 \mathrm{~A} \quad 2.3012(14)$ & C1B C2B $1.521(2)$ \\
\hline O1B C1B 1.249(2) & C8B C7B 1.519(2) \\
\hline O1A C1A $1.253(2)$ & C7A C8A $1.520(2)$ \\
\hline O4A C8A $1.293(2)$ & C11B C12B 1.400(2) \\
\hline O2A C4A $1.304(2)$ & C11B C16B 1.393(2) \\
\hline O6A C10A 1.232(2) & С10В С9B 1.523(2) \\
\hline O4B C8B 1.278(2) & C10AC9A 1.522(2) \\
\hline O2B C4B 1.305(2) & C1A C2A $1.514(2)$ \\
\hline O6B C10B 1.225(2) & С12B C13B 1.397(2) \\
\hline O5A C8A $1.228(2)$ & C6A C5A $1.516(2)$ \\
\hline O5B C8B 1.238(2) & C12AC11A 1.399(2) \\
\hline O3A C4A $1.215(2)$ & C12AC13A 1.393(2) \\
\hline O3B C4B 1.221(2) & C4A C3A 1.534(2) \\
\hline N4A C10A 1.342(2) & C16AC11A 1.395(2) \\
\hline N4A C11A $1.420(2)$ & C16AC15A 1.384(3) \\
\hline N4B C11B 1.417(2) & C16B C15B 1.385(3) \\
\hline N4B C10B 1.350(2) & C13AC14A 1.384(3) \\
\hline N1B C1B $1.319(2)$ & C14B C13B 1.378(2) \\
\hline N1B C12B 1.423(2) & C14B C15B 1.387(3) \\
\hline N3A C7A $1.486(2)$ & C5B C6B 1.522(3) \\
\hline N3A C6A $1.500(2)$ & C15AC14A 1.388(3) \\
\hline N3A C9A $1.502(2)$ & C4B C3B 1.532(2) \\
\hline
\end{tabular}

Table S11 Bond Angles for $\left\{[\mathrm{Fe}(\text { edtaod })]_{2} \mathrm{O}\right\} \cdot 4 \cdot 4 \mathrm{H}_{2} \mathrm{O}$.

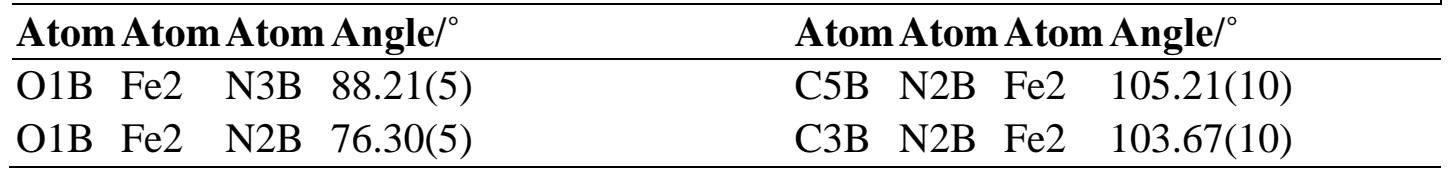




\begin{tabular}{|c|c|c|c|}
\hline O4B & $\mathrm{Fe} 2$ & O1B $159.20(5)$ & C3B N2B C2B $110.44(14)$ \\
\hline O4B & $\mathrm{Fe} 2$ & N3B 78.94(5) & C3B N2B C5B 113.78(13) \\
\hline O4B & $\mathrm{Fe} 2$ & N2B $85.23(5)$ & O1B C1B N1B 122.97(15) \\
\hline O9 & $\mathrm{Fe} 2$ & O1B 96.57(5) & O1B C1B C2B 119.06(15) \\
\hline O9 & $\mathrm{Fe} 2$ & O4B 101.86(5) & N1B C1B C2B 117.97(15) \\
\hline O9 & $\mathrm{Fe} 2$ & O2B $102.43(5)$ & O4B C8B C7B 115.44(15) \\
\hline O9 & $\mathrm{Fe} 2$ & N3B 101.01(5) & O5B C8B O4B 125.02(17) \\
\hline O9 & $\mathrm{Fe} 2$ & N2B $172.86(5)$ & O5B C8B C7B 119.52(17) \\
\hline $\mathrm{O} 2 \mathrm{~B}$ & $\mathrm{Fe} 2$ & O1B 92.41(5) & N3A C7A C8A 113.70(13) \\
\hline $\mathrm{O} 2 \mathrm{~B}$ & $\mathrm{Fe} 2$ & O4B 92.94(5) & C12B C11B N4B $123.37(15)$ \\
\hline $\mathrm{O} 2 \mathrm{~B}$ & $\mathrm{Fe} 2$ & N3B $156.32(5)$ & C16B C11B N4B $117.87(15)$ \\
\hline $\mathrm{O} 2 \mathrm{~B}$ & $\mathrm{Fe} 2$ & N2B $77.75(5)$ & C16B C11B C12B 118.75(16) \\
\hline N3B & $\mathrm{Fe} 2$ & N2B $79.42(5)$ & O6B C10B N4B $123.15(16)$ \\
\hline O1A & Fe1 & N3A 90.93(5) & O6B C10B C9B $121.43(15)$ \\
\hline O1A & Fe1 & N2A $76.41(5)$ & N4B C10B C9B $115.41(14)$ \\
\hline $\mathrm{O} 4 \mathrm{~A}$ & Fe1 & O1A 161.71(5) & O6A C10AN4A 123.22(15) \\
\hline O4A & Fe1 & N3A $78.95(5)$ & O6A C10AC9A 120.86(15) \\
\hline $\mathrm{O} 4 \mathrm{~A}$ & $\mathrm{Fe} 1$ & N2A $86.52(5)$ & N4A C10AC9A 115.92(14) \\
\hline $\mathrm{O} 2 \mathrm{~A}$ & $\mathrm{Fe} 1$ & O1A 91.04(5) & O1A C1A N1A $122.00(15)$ \\
\hline $\mathrm{O} 2 \mathrm{~A}$ & Fe1 & O4A 91.66(5) & O1A C1A C2A 118.74(15) \\
\hline $\mathrm{O} 2 \mathrm{~A}$ & Fe1 & N3A $154.20(5)$ & N1A C1A C2A $119.25(15)$ \\
\hline $\mathrm{O} 2 \mathrm{~A}$ & Fe1 & N2A $77.50(5)$ & C11B C12B N1B $124.76(15)$ \\
\hline O9 & Fe1 & O1A $93.25(5)$ & C13B C12B N1B $115.72(15)$ \\
\hline O9 & Fe1 & O4A $103.61(5)$ & C13B C12B C11B 119.42(15) \\
\hline O9 & Fe1 & O2A $104.15(5)$ & N3A C6A C5A 112.15(13) \\
\hline O9 & Fe1 & N3A $101.41(5)$ & O4A C8A C7A 115.90(14) \\
\hline O9 & Fe1 & N2A $169.60(5)$ & O5A C8A O4A $124.73(16)$ \\
\hline N3A & Fe1 & N2A $77.99(5)$ & O5A C8A C7A 119.32(15) \\
\hline $\mathrm{C} 1 \mathrm{~B}$ & O1B & $\mathrm{Fe} 2 \quad 121.83(11)$ & C11AC12AN1A 124.31(15) \\
\hline $\mathrm{C} 1 \mathrm{~A}$ & O1A & Fe1 120.62(11) & C13AC12AN1A 116.21(16) \\
\hline C8A & O4A & $119.80(11)$ & C13AC12AC11A 119.47(16) \\
\hline C4A & $\mathrm{O} 2 \mathrm{~A}$ & $120.77(11)$ & O2A C4A C3A 115.63(15) \\
\hline C8B & O4B & $117.72(11)$ & O3A C4A O2A 124.12(17) \\
\hline Fe1 & O9 & $150.80(7)$ & O3A C4A C3A 120.18(16) \\
\hline C4B & $\mathrm{O} 2 \mathrm{~B}$ & $\mathrm{Fe} 2 \quad 119.87(11)$ & C15AC16AC11A 121.01(17) \\
\hline C10A & N4A & C11A 124.47(14) & C12AC11AN4A $123.37(15)$ \\
\hline C10B & N4B & C11B 122.63(14) & C16AC11AN4A $117.48(16)$ \\
\hline $\mathrm{C} 1 \mathrm{~B}$ & N1B & C12B 127.09(14) & C16AC11AC12A 119.01(16) \\
\hline C7A & N3A & Fe1 103.00(9) & C15B C16B C11B 121.06(16) \\
\hline C7A & N3A & C6A 109.26(12) & N2B C2B C1B 109.53(14) \\
\hline C7A & N3A & C9A 106.11(12) & N3A C9A C10A 112.73(13) \\
\hline C6A & N3A & Fe1 109.44(10) & C14AC13AC12A 120.90(17) \\
\hline
\end{tabular}




\begin{tabular}{|c|c|c|c|c|}
\hline C6A & N3A & C9A & $110.78(12)$ & C13B C14B C15B 119.68(16) \\
\hline C9A & $\mathrm{N} 3 \mathrm{~A}$ & Fe1 & $117.68(10)$ & N2A C5A C6A $108.85(13)$ \\
\hline C9B & N3B & $\mathrm{Fe} 2$ & 119.01(10) & C14B C13B C12B 120.93(16) \\
\hline C6B & N3B & $\mathrm{Fe} 2$ & $109.79(11)$ & N3B C9B C10B 113.60(14) \\
\hline C6B & $\mathrm{N} 3 \mathrm{~B}$ & C9B & $110.00(13)$ & N2A C2A C1A $109.07(13)$ \\
\hline C7B & N3B & $\mathrm{Fe} 2$ & $101.11(10)$ & N2B C5B C6B 109.54(14) \\
\hline C7B & N3B & C9B & $106.43(14)$ & N3B C6B C5B 113.44(14) \\
\hline C7B & N3B & C6B & $109.87(13)$ & C16AC15A C14A 119.85(17) \\
\hline C1A & N1A & $\mathrm{C} 12 \mathrm{~A}$ & $123.13(14)$ & C16B C15B C14B 119.85(16) \\
\hline $\mathrm{C} 5 \mathrm{~A}$ & $\mathrm{~N} 2 \mathrm{~A}$ & $\mathrm{Fe} 1$ & $106.35(10)$ & O2B C4B C3B 114.24(15) \\
\hline $\mathrm{C} 2 \mathrm{~A}$ & $\mathrm{~N} 2 \mathrm{~A}$ & $\mathrm{Fe} 1$ & $109.27(10)$ & O3B C4B O2B 124.53(17) \\
\hline $\mathrm{C} 2 \mathrm{~A}$ & $\mathrm{~N} 2 \mathrm{~A}$ & $\mathrm{C} 5 \mathrm{~A}$ & $111.62(13)$ & O3B C4B C3B 121.22(16) \\
\hline $\mathrm{C} 3 \mathrm{~A}$ & $\mathrm{~N} 2 \mathrm{~A}$ & $\mathrm{Fe} 1$ & $103.59(10)$ & N2A C3A C4A 110.81(14) \\
\hline $\mathrm{C} 3 \mathrm{~A}$ & $\mathrm{~N} 2 \mathrm{~A}$ & $\mathrm{C} 5 \mathrm{~A}$ & $113.68(13)$ & N2B C3B C4B 108.64(13) \\
\hline $\mathrm{C} 3 \mathrm{~A}$ & $\mathrm{~N} 2 \mathrm{~A}$ & $\mathrm{C} 2 \mathrm{~A}$ & $111.78(13)$ & C13AC14A C15A 119.67(17) \\
\hline $\mathrm{C} 2 \mathrm{~B}$ & $\mathrm{~N} 2 \mathrm{~B}$ & $\mathrm{Fe} 2$ & $110.63(10)$ & N3B C7B C8B 110.69(15) \\
\hline $\mathrm{C} 2 \mathrm{~B}$ & $\mathrm{~N} 2 \mathrm{~B}$ & $\mathrm{C} 5 \mathrm{~B}$ & $112.60(14)$ & \\
\hline
\end{tabular}

Table S12 Hydrogen Atom Coordinates $\left(\AA \times 10^{4}\right)$ and Isotropic Displacement Parameters $\left(\AA^{2} \times 10^{3}\right)$ for $\left\{[\mathrm{Fe}(\text { edtaod })]_{2} \mathrm{O}\right\} \cdot 4.4 \mathrm{H}_{2} \mathrm{O}$.

\begin{tabular}{lllll|}
\hline Atom & $\boldsymbol{x}$ & $\boldsymbol{y}$ & $\boldsymbol{z}$ & $\mathbf{U}(\mathbf{e q )}$ \\
\hline H3WA & 2199.62 & 1250.13 & 1833.34 & 32 \\
H3WB & 1573.21 & 843.82 & 1957.68 & 32 \\
H2WA & 2953.72 & -228.82 & 1095.19 & 40 \\
H2WB & 2679.5 & 174.29 & 1558.07 & 40 \\
H1WA & 4713.11 & 151.96 & 1711.27 & 35 \\
H1WB & 3979.19 & 262.92 & 1570.12 & 35 \\
H4A & 2426.09 & 2389.83 & 3177.74 & 15 \\
H4B & 4862.14 & 2391.01 & 4287.8 & 14 \\
H1B & 3240.24 & 714.99 & 5004.13 & 14 \\
H1A & 4203.34 & 1063.66 & 2313.19 & 16 \\
H7AA & 3021.13 & 4532.17 & 2539.61 & 14 \\
H7AB & 3446.03 & 4520.44 & 1925.65 & 14 \\
H6AA & 3855.75 & 3554.81 & 1519.09 & 15 \\
H6AB & 3902.44 & 2752.04 & 1816.07 & 15 \\
H16A & 1746.8 & 1233.56 & 3460.33 & 20 \\
H16B & 5769.68 & 1350.81 & 4049.73 & 18 \\
H2BA & 1972.24 & 1508.47 & 4847.85 & 20 \\
H2BB & 2457.5 & 1641.75 & 5426.81 & 20 \\
H9AA & 2539.52 & 3429.62 & 2010.74 & 14 \\
\hline
\end{tabular}




\begin{tabular}{lllll}
\hline H9AB & 2509.01 & 3449.21 & 2710.18 & 14 \\
H13A & 3914.58 & -33.36 & 2971.41 & 20 \\
H14B & 4960.87 & -735.99 & 4156.6 & 20 \\
H5AA & 5112.54 & 3169.57 & 1635.69 & 15 \\
H5AB & 4956.2 & 3922.86 & 1989.17 & 15 \\
H13B & 3850.98 & -273.8 & 4523.92 & 17 \\
H9BA & 4621.67 & 3338.27 & 5488.62 & 17 \\
H9BB & 4640.91 & 3396.41 & 4790.76 & 17 \\
H2AA & 5457.83 & 1967.52 & 2647.85 & 18 \\
H2AB & 4992.99 & 2051.77 & 2052.64 & 18 \\
H5BA & 2145.54 & 2697.24 & 5887.11 & 19 \\
H5BB & 2132.19 & 3482.18 & 5556.7 & 19 \\
H6BA & 3294.99 & 3367.57 & 5987.54 & 21 \\
H6BB & 3405.33 & 2555.19 & 5720.54 & 21 \\
H15A & 1913.21 & 14.07 & 3768.2 & 23 \\
H15B & 5904.55 & 84.54 & 3879.94 & 21 \\
H3AA & 5870.94 & 3778.43 & 2629.31 & 19 \\
H3AB & 6175.79 & 2945.63 & 2581.31 & 19 \\
H3BA & 1262.3 & 3212.12 & 4889.62 & 21 \\
H3BB & 1103.51 & 2340.67 & 4961.77 & 21 \\
H14A & 2992.46 & -629.88 & 3509.08 & 23 \\
H7BA & 3942.89 & 4409.54 & 4884.61 & 20 \\
H7BB & 3713.64 & 4356.27 & 5560.82 & 20 \\
H5WA & 4367.1 & 6032.18 & 4095.39 & 53 \\
H5WB & 4547.72 & 5317.77 & 4316.66 & 53 \\
H4WA & 5652.56 & 4369.69 & 4674.2 & 64 \\
H4WB & 5197.03 & 4189.99 & 4186.32 & 64 \\
\hline
\end{tabular}

Table S13 Atomic Occupancy for $\left\{[\mathrm{Fe}(\text { edtaod })]_{2} \mathrm{O}\right\} \cdot 4.4 \mathrm{H}_{2} \mathrm{O}$.

\begin{tabular}{|c|c|c|c|}
\hline Atom & Occupancy & Atom Occupancy & Atom Occupancy \\
\hline $\mathrm{O5W}$ & 0.7 & H5WA 0.7 & H5WB 0.7 \\
\hline $\mathrm{O} 4 \mathrm{~W}$ & 0.7 & H4WA 0.7 & H4WB 0.7 \\
\hline
\end{tabular}

